\title{
Experimental investigations of graded sediment transport under unsteady flow hydrographs
}

\section{Citation for published version:}

Wang, L, Cuthbertson, A, Pender, G \& Cao, Z 2015, 'Experimental investigations of graded sediment transport under unsteady flow hydrographs', International Journal of Sediment Research, vol. 30, no. 4, pp. 306-320. https://doi.org/10.1016/j.ijsrc.2015.03.010

\section{Digital Object Identifier (DOI):}

10.1016/j.ijsrc.2015.03.010

\section{Link:}

Link to publication record in Heriot-Watt Research Portal

\section{Document Version:}

Peer reviewed version

\section{Published In:}

International Journal of Sediment Research

\section{General rights}

Copyright for the publications made accessible via Heriot-Watt Research Portal is retained by the author(s) and / or other copyright owners and it is a condition of accessing these publications that users recognise and abide by the legal requirements associated with these rights.

\section{Take down policy}

Heriot-Watt University has made every reasonable effort to ensure that the content in Heriot-Watt Research Portal complies with UK legislation. If you believe that the public display of this file breaches copyright please contact open.access@hw.ac.uk providing details, and we will remove access to the work immediately and investigate your claim. 


\section{Experimental Investigations of Graded Sediment Transport under Unsteady Flow Hydrographs}

Le Wang ${ }^{1}$, Alan J S Cuthbertson ${ }^{2, *}$, Gareth Pender ${ }^{3}$ and Zhixian Cao ${ }^{4 a, b}$

${ }^{1} \mathrm{PhD}$ student, Institute for Infrastructure and Environment, Heriot-Watt University, Edinburgh EH14 4AS, UK. Email: $\underline{1 w 151 @ h w . a c . u k ~}$

${ }^{2}$ Associate Professor, Institute for Infrastructure and Environment, Heriot-Watt University, Edinburgh EH14 4AS, UK. E-mail: a.cuthbertson@hw.ac.uk

${ }^{3}$ Professor, Institute of Infrastructure and Environment, Heriot-Watt University, Edinburgh EH14 4AS, UK. E-mail: g.pender@hw.ac.uk

${ }^{4}$ Professor, ${ }^{\mathrm{a}}$ Institute for Infrastructure and Environment, Heriot-Watt University, Edinburgh, UK; Professor, ${ }^{\mathrm{b}}$ State Key Laboratory of Water Resources and Hydropower Engineering Science, Wuhan University, Wuhan, People's Republic of China. Email: z.cao@hw.ac.uk; zxcao@whu.edu.cn

* Corresponding author. 


\title{
Experimental Investigations of Graded Sediment Transport under Unsteady Flow Hydrographs
}

\begin{abstract}
Natural fluvial channels can experience significant variations in sediment transport rates under unsteady flow conditions, especially during flood hydrograph events. At present, however, there is a distinct lack of understanding of the interaction between unsteady hydrograph flow properties and temporal variability in graded sediment transport rates. In the current study, a series of parametric experiments was conducted to investigate the response of two graded sediment beds to a range of different unsteady hydrograph flow conditions. Investigations of the total and fractional bed-load sediment transport rates revealed strong temporal variations in transport over the hydrographs, with size-dependent temporal lag effects observed between peak flow conditions and peak bed-load transport rates. Specifically, coarse gravels had increased mobility during the rising limb of the hydrographs, attaining their peak bed-load transport rate either prior to, or near, peak flow conditions. By contrast, the finer grades tended to have enhanced mobility during the receding limb of the hydrographs, with peak transport rates measured after peak flow conditions had passed. Grain size distributions measured from the collected bed-load samples also indicated material coarsening over the rising limb and fining during the receding limb, while corresponding image analysis measurements of bed surface composition showed only marginal variation over the hydrographs. Computation of total and fractional sediment yields revealed that the bimodal sediment mixture tested was transported at significantly higher rates than the unimodal mixture over all hydrograph conditions tested. This finding indicated that the unimodal sediment bed was inherently more stable than the bimodal bed due to the increased abundance of medium-sized gravels present in the uni-modal sediment grade. The parametric
\end{abstract}


dependences established in the study have clear implications for improved understanding of fractional inter-granular effects at the bed surface and their influence on graded sediment transport processes within natural fluvial channels under flood flow hydrographs.

Keywords: Graded sediments, bed-load transport, unsteady flow hydrographs, temporal lag, fractional mobility.

\section{Introduction}

Within natural fluvial systems, unsteady flood flow hydrographs and graded sediment transport are known to be inherently linked, with the occurrence of annual bulk sediment transport generally concentrated during flood events (e.g., Phillips and Sutherland., 1990; Berta et al., 2010). Investigations of bed-load transport under such unsteady flow conditions began in the 1980s with much attention initially focused on field observations. Unfortunately, accurate measurements of sediment transport rates during high flow conditions are extremely difficult to obtain (e.g., Graf and Qu, 2004; Mao, 2012) and, thus, laboratory experiments have become increasingly important in the attempt to understand the dominant physical interactions between unsteady flow hydrographs and bed-load sediment transport. However, the majority of these investigations have adopted idealized representations of the flood hydrograph conditions, either by modelling temporal variability in flow rates through a series of incremental steady-state flows (e.g., Parker et al., 2007; Piedra, 2010) or through the selection of a constant rate of change of flow rate during the rising and receding hydrograph limbs (i.e. specification of triangular or trapezoidal-shaped hydrographs) (e.g., Lee et al., 2004; Bombar et al., 2011). Thus, none of these experimental configurations have, to-date, represented fully the strong temporal variability associated with naturally-shaped flood hydrographs or revealed the underlying time-dependent mechanisms associated with the 
response of graded sediment beds to these changing flow conditions. For the wide grain size ranges typically encountered in natural river beds (i.e. fine sands to coarse gravels), this has meant that knowledge of graded sediment transport during flood events has remained limited.

Noticeable temporal lag effects between measured peak bed-load transport rates and peak unsteady flow conditions have been widely reported to arise from the interaction and response of graded sediments to time-varying hydraulic parameters at the bed surface. Experiments by Griffiths (1976) and Bell (1980) revealed that temporal lag effects for bedload transport occurred under flood waves. Similarly, Phillips and Sutherland (1990) related the inertial response of alluvial systems to varying flow conditions to formulate a temporal lag model. More recently, experimental observations by Bombar et al. (2011) indicated that the average time lag between the peak flow and maximum bed-load transport rate varied depending on the shape of the idealized hydrograph tested (i.e. triangular- and trapezoidalshaped hydrographs). As yet, however, there remains no detailed study into graded sediment transport under natural-shaped, flood hydrographs and, consequently, knowledge of the interactions between different size classes within sediment beds, and their relative abundance in bed-load transport at different periods during the hydrograph also remains unclear.

A number of non-dimensional parameters have been introduced to characterize the unsteady properties of flow hydrographs (e.g. Graf and Suszka, 1985; Yen and Lee, 1995). However, the individual and combined effect of these parameters on bed-load sediment transport yields has not, as yet, been studied in a systematic manner over a range of hydrograph conditions. Within the current study, a series of parametric experiments, was therefore, conducted to investigate systematically the temporal variability of graded bed-load sediment transport processes and bed surface composition associated with the passage of such flow hydrographs. 


\section{Experimental Arrangement}

\subsection{Flume Set-up and Procedure}

The experimental studies were performed in an $8 \mathrm{~m}$ long, $0.3 \mathrm{~m}$ wide, and $0.3 \mathrm{~m}$ deep flowrecirculating flume set at a constant bed slope of 0.0083 (1:120) (see Figure 1). The flume was equipped with a computer-controlled pump invertor capable of producing controlled, unsteady flow conditions within the channel with flow rates up to $201 \mathrm{~s}^{-1}$. These flows were measured in the delivery pipe by a non-intrusive ultrasonic flow meter to an accuracy of $\pm 0.01 \mathrm{~s}^{-1}$. This automated unsteady flow system was utilized to generate different naturalshaped hydrograph flow profiles with different magnitudes and shape characteristics along the flume channel.

The initial bed configuration within the channel consisted of an erodible, graded sediment layer of uniform thickness $\sim 8 \mathrm{~cm}$, placed along the full working length of the flume channel. This bed layer was scraped flat using a screed board, which ran along the full length of the flume on two parallel carriage rails. Measurements of the initial bed surface elevation were taken with an electronic pointer gauge (to $\pm 1 \mathrm{~mm}$ accuracy) to ensure the required slope, $S_{0}$, was achieved. It should also be noted that no sediment feed was introduced at the upstream end of the flume during any of the tests.

Prior to each test, the downstream tailgate was fully raised and water levels within the sump tanks checked. The flow-recirculation pump was then switched on and set to an initially low flow rate to ensure minimal disturbance of the bed surface structure during the initial wetting stage. Once the sediment bed was fully submerged, the flow rate was programmed to increase gradually to an established sub-threshold, antecedent (base) flow condition $\left(Q_{0}=81 \mathrm{~s}^{-1}\right)$. 
During the establishment of antecedent flow, the flume tailgate was lowered to a predefined position to diminish backwater effects and develop steady, uniform flow conditions along the channel. The water surface elevation was measured and checked by the electronic pointer gauge along the flume working section to ensure uniform flow conditions were indeed achieved. This base flow condition was designed to rework the graded sediment mixtures in the test section (i.e. upstream of the sediment trap) for an extended period of up to 1 hour, without mobilizing the bed layer, to ensure that all experimental runs were conducted under consistent initial bed conditions.

Following the period of antecedent flow, the computer-controlled frequency inverter was programmed to smoothly adjust the pump speed and, hence, achieve the required temporallyvarying flow profiles for the range of design hydrographs tested. During these unsteady flow conditions, temporal variations in the water surface elevation were measured at 10 minute intervals along the working section by the electronic pointer gauge. Although the tailgate was not adjusted during this unsteady period, flow conditions were typically found to be nearuniform during the hydrographs, with only small variations in water surface elevations (up to a few $\mathrm{mm}$ ) in most cases and measured water surface slopes, $S_{f} \approx S_{0}$. Additionally, as the rate of change in flow rate $d Q / d t$ was relatively low for all design hydrographs tested, any spatial variations in these measured water surface elevations along the channel due directly to the flow unsteadiness were deemed negligible.

These findings were confirmed through calculations of the global bed shear stress, which was predicted for the steady, uniform flow base flow conditions by the equation (Shvidchenko and Pender, 2000; Shvidchenko et al.,2001):

$\tau=\rho_{f} g R_{b} S_{0}$ 
where $\rho_{f}$ is the fluid density, $g$ is the acceleration due to gravity and $R_{b}$ is the hydraulic radius of the bed. Corresponding shear stresses during the unsteady flow hydrographs were predicted by a new method based on the Saint-Venant equations (Ghimire and Deng, 2012), i.e:

$$
\tau=\rho_{f} g R_{b}\left(S_{f}+\alpha \frac{\partial Q}{\partial t}\right)
$$

where $\alpha\left[=1 /\left(B C_{w}{ }^{2}\right)\right]$ is a parameter relating the flood hydrograph slope with shear stress $\tau$ during unsteady flow [in which $B$ is the channel width, $C_{w}=(g h)^{1 / 2}$ is the average wave celerity and $h$ is the flow depth]; $Q$ is the flow rate and $t$ is the time. Thus, for mildly unsteady flows ( $d Q / d t \rightarrow 0)$, Eq. (2) becomes equivalent to the shear stress for steady, uniform flow conditions (i.e. when water surface slope $S_{f}=S_{0}$ ). In the current study, this was indeed found to be the case (i.e. $S_{f} \approx S_{0}$ ) and predictions from both Eqs. 1 and 2 were found to be in close agreement for the range of unsteady flow hydrograph conditions considered [with $\alpha=4.25-7.39\left(\mathrm{~m}^{-3} \mathrm{~s}^{2}\right)$ and $d Q / d t \sim O(10)^{-6}\left(\mathrm{~m}^{3} \mathrm{~s}^{-2}\right)$, typically].

\subsection{Bed Sediment Properties}

In order to create the design sediment mixtures for the current tests, natural sands and gravels ranging from $d=1 \mathrm{~mm}$ to $16 \mathrm{~mm}$ were sieved into eight individual size fractions. These fractions were combined in required proportions to generate two distinct sediment mixtures, both having the same median size, $d_{50}=5.0 \mathrm{~mm}$, and geometric standard deviation, $\sigma_{\mathrm{g}}=$ 1.89, but with uni-modal (UM) and bimodal (BM) particle size distributions (see Figure 2). A summary of the main percentile sizes for these design sediment mixtures is also given in Table 1. 
The study also employed a classification system proposed by Frey et al. (2003), whereby the graded sediments were split into three classes (i.e. fine, medium, and coarse) depending on grain diameter. This was done to aid description and analysis of the fractional composition of bed-load transport generated under the unsteady flow hydrographs. The grain sizes for these defined size classes were the same for both the UM and BM sediment mixtures (see Table 1), while the relative proportions of these fine, medium and coarse size classes in the undisturbed test bed sediment layers were (i) UM grade: 29, 41.2, and 29.8\%, respectively, and (ii) BM grade: $38.9,22.3$, and $38.8 \%$, respectively.

\subsection{Experimental Measurements}

Bed-load sediment transport was measured within the channel using a demountable sediment trap located at the end of the test bed section, $2.5 \mathrm{~m}$ from the downstream end of the flume (see Figure 1). Bed-load sediment samples were collected in the trap at 10 minute intervals over the duration of the hydrographs. This sampling period was considered sufficiently long to reduce variability associated with individual sediment flux measurement (i.e. by averaging out larger transport fluctuations that occur during the sampling period). The sediment trap itself consisted of inter-changeable transparent Perspex sediment collection boxes (370 mmlong by $150 \mathrm{~mm}$-wide by $30 \mathrm{~mm}$-deep), an isolation valve, two bleed valves and a jack (see Figure 3). A small upper Perspex box was also positioned and fixed above the slot such that it was flush with the bed surface layer of the erodible sediment. Prior to the start of the bed-load measurements, one of the sediment collection boxes was positioned beneath the slot cut in the flume bed and jacked up firmly against the isolation valve, providing a seal against the neoprene rim. The collection box was then filled with water (via the water injection valve), with bleed valves used to eliminate air bubbles from the trap system prior to opening of the isolation valve. During the subsequent 10 minute bed-load measurement period, transported 
sediment samples accumulated in the collection box. At the end of this period, the collection box was again isolated from the upper trap section by closure of the valve before being removed and replaced by the second collection box for the subsequent bed-load measurement period. Individual sediment samples collected from the boxes were subsequently dried in an oven, weighed, and sieved to obtain time-averaged total, $q_{b}$, and fractional, $q_{b i}$, bed-load transport rates for each 10 minute bed-load sampling period.

Temporal changes in the bed surface composition were also monitored at equivalent 10 minute intervals throughout each hydrograph using a vertically-mounted digital camera and Perspex window arrangement that allowed the bed to be visualized through the water surface. In this way, high-resolution images of the sediment bed surface were obtained at a bed location adjacent to the sediment trap for particle size analysis using the Sedimetrics Digital Gravelometer software package (Graham et al., 2005) (see example images in Figure 4). The bed section used for this visualization technique was located $0.5 \mathrm{~m}$ upstream of the sediment trap (Figure 1). In utilizing this technique, accurate measurements of bed surface grain size distributions relied on selection of an appropriate bed imaging size, based on the camera resolution $(18 \mathrm{MP})$ and smallest grain size $(1 \mathrm{~mm})$. The selected bed image size $(0.2 \mathrm{~m} \times 0.15$ m) resulted in an overall image pixel resolution of 1 pixel $\approx 40 \mu \mathrm{m}$, which was significantly finer than the smallest grain size $\left(d_{i}=1 \mathrm{~mm}\right)$. In addition, it was anticipated that measuring bed surface composition over a relatively large bed area (relative to the required resolution) would limit variability in surface composition measurements that might have been generated for a smaller bed area. Calibration of the bed imaging technique was conducted under static bed conditions by comparing grain size distributions obtained from high-resolution images of both UM and BM sediment mixtures with corresponding distributions obtained by carefully sampling of the bed surface layer (i.e. collection, drying, sieving, and weighing). Figure 5 
shows that these grain size distributions are in reasonable agreement, although the bed imaging technique was found to typically over-predict the proportion of finer sediments in the bed surface layer, and, thus, under-predicting the medium to coarse size fractions, compared with the direct sampling measurements. However, this discrepancy may also have resulted from the loss of a proportion of the finer fractions during the direct sampling procedure. It is interesting to note that the measured grain size distributions at the bed surface are typically coarser (i.e. $d_{s 50}=7-8 \mathrm{~mm}$, Figure 5 , where $s$ denotes the surface sediment) than the respective $\mathrm{UM}$ and $\mathrm{BM}$ sediment mixtures (i.e. $d_{50}=5 \mathrm{~mm}$, Figure 2). This bed surface coarsening effect is discussed in greater detail in Section 4.1 of the paper.

\subsection{Design Hydrographs}

Two sets of design hydrographs (i.e. HY1_1 and HY1_2, Figures $6 a$ and $6 \mathrm{~b}$ and Table 2) were tested during the flume experiments. The first set (Figure 6a) had the same fixed overall duration $\left(T_{h}=7200 \mathrm{~s}\right)$ and total hydrograph water volume $\left(V_{h}=39.26 \mathrm{~m}^{3}\right.$, excluding base flow), but varying rising and receding limb profiles to test the parametric influence of hydrograph shape on the fractional bed-load transport response. Specifically, HY1_1a considered symmetrical conditions with equal duration rising and receding limbs, while HY1_1b and HY1_1c represented asymmetric conditions with relatively shorter and longer duration rising limbs, respectively, compared to the receding limb durations (i.e. shorter and longer time to peak flow). In addition, an equivalent-volume steady flow condition (see ES in Figure 6a and Table 2) was also tested over the same duration (7200 s) to highlight the difference in sediment transport yields obtained under the unsteady flow hydrographs and equivalent, steady flow conditions. The second set of hydrographs tested (i.e. HY1_2, Figure $6 \mathrm{~b}$ and Table 2$)$ were all symmetrical, but had variable overall water volumes $\left(V_{h}=27.8 \rightarrow\right.$ $\left.39.26 \mathrm{~m}^{3}\right)$, peak flow magnitudes $\left(Q_{\text {peak }}=14 \rightarrow 181 \mathrm{~s}^{-1}\right)$, and overall durations $\left(T_{h}=7200 \rightarrow\right.$ 
$8460 \mathrm{~s}$ ) to determine the influence of hydrograph magnitude and unsteadiness on the fractional bed-load transport response.

Continuous monitoring of the actual flow rate entering the flume channel by the ultrasonic flow meter attached to the upstream supply pipe indicated slight deviations from these design hydrograph flow conditions. However, the measured standard deviations of up to $0.1781 \mathrm{~s}^{-1}$ (i.e. approximately $2.2 \%$ of the base flow conditions) were deemed negligible.

\section{Scaling Considerations}

In order to permit direct comparison of results from both experimental series, the discharge, length, and velocity scales associated with the initial, antecedent flow conditions (i.e. flow rate, $Q_{0}$, flow depth, $h_{0}$, and average flow velocity, $u_{0}$ ) were used, along with channel width, $B$, and sediment properties (i.e. density, $\rho_{s}$, and representative median, $d_{50}$, and fractional, $d_{i}$, grain sizes) to non-dimensionalize the experimental time scale, $t$, unsteady flow rates, $Q$, and total and fractional bed-load sediment transport rates, $q_{b}$, and, $q_{b i}$, respectively, such that:

$$
\begin{aligned}
& t^{*}=t\left(\frac{u_{0}}{h_{0}}\right) \\
& Q^{*}=\frac{Q}{Q_{0}}=\frac{Q}{u_{0} h_{0} B} \\
& q_{b}^{*}=\frac{q_{b}}{\rho_{s} \sqrt{(s-1) g d_{b 50}^{3}}} ; q_{b i}^{*}=\frac{q_{b i}}{f_{i} \rho_{s} \sqrt{(s-1) g d_{i}^{3}}}
\end{aligned}
$$

where $s=\rho_{s} / \rho_{f}$ is submerged specific gravity of sediment. Note that in Eq. 3(c), individual $d_{b 50}$ values were calculated for each trapped bed-load sample collected during the runs, while corresponding proportions $f_{i}$ for the specific size fractions, $i$, in the bed surface layer were measured visually (using Sedimetrics) during the same sample periods (e.g. Parker, 1990). These values of $d_{b 50}$ and $f_{i}$, thus, allow the non-dimensional transport rates, $q_{b}{ }^{*}$ and $q_{b i}{ }^{*}$, [Eq. 
3(c)] to be localized in time, relevant to specific particle size distributions of individual collected trap samples, as well as the corresponding instantaneous bed surface composition.

Based on previous studies (e.g., Lee et al., 2004; Graf and Qu, 2004; Bombar et al., 2011), specific unsteady flow characteristics expected to have significant influence on bed-load sediment transport and bed surface composition include (i) the hydrograph unsteadiness (or flashiness), (ii) the overall volume (or magnitude) of the hydrograph, and (iii) the hydrograph shape. Thus, for wider investigation of these parametric influences, it is required to develop appropriate non-dimensional parameters describing these unsteady flow characteristics and the resulting response of the sediment bed. The independent variables in the problem can be subdivided (Lee et al., 2004) into: (i) fluid properties (i.e. dynamic viscosity, $\mu$, and density, $\rho_{f}$ ); (ii) channel geometry (i.e. width, $B$, and slope, $S_{0}$ ); (iii) sediment properties (i.e. density, $\rho_{s}$, and representative grain size, $d$ ); (iv) hydraulic flow characteristics [i.e. depth, $h$, shear velocity, $u *$, and discharge volume under the hydrograph, $V_{h}$ (excluding the base flow)]; and (v) time (i.e. hydrograph duration, $T_{h}$ ). Thus, a dimensional functional relationship can be written for the overall sediment transport yield, $W_{t}(\mathrm{~kg})$, over the hydrograph duration in the form:

$$
W_{t}=f\left(B, S_{0}, \rho_{f}, \mu, g, \rho_{s}, d, h, u_{*}, V_{h}, T_{h}, T_{r}, T_{f}\right)
$$

where $T_{r}$ and $T_{f}$ are the respective durations of the rising and receding hydrograph limbs (i.e. $\left.T_{h}=T_{r}+T_{f}\right)$. Clearly, the parameters $h$ and $u *$ are time dependent, but within the context of the total sediment yield, $W_{t}$, over the duration of the hydrograph, it might be expected that a reference or integral value for both these parameters be adopted in Eq. (4). If it is assumed that fluid properties, channel geometry, and sediment density remain unchanged during the study, an appropriate non-dimensional functional relationship can be derived from dimensional analysis considerations such that: 


$$
W_{t}^{*}=f\left(\frac{h}{u_{*} T_{h}} ; \frac{V_{h} u_{*}^{2}}{g h^{3} B} ; \frac{T_{r}}{T_{f}}\right)
$$

where $W_{t}^{*}$ is the normalized sediment transport yield over the hydrograph duration.

Following Bombar et al. (2011), the non-dimensional total and fractional sediment transport yields $\left(W_{t}^{*}\right.$ and $W_{t, i}{ }^{*}$, respectively) can be defined as:

$$
W_{t}^{*}=\frac{W_{t}}{\rho_{s} b d_{50}^{2}} ; W_{t, i}^{*}=\frac{W_{t, i}}{\rho_{s} b d_{i}^{2}}
$$

where $W_{t, i}$ is the fractional transport yield $(\mathrm{kg})$ over the hydrograph duration; $b$ is the sediment trap width $(<B) ; d_{50}$ is the median grain size of the original sediment mix; and $d_{i}$ is the representative grain size of individual size fractions. In relation to the general parametric relationship given by Eq. (5), the first non-dimensional parameter on the right hand side describes the influence of flow depth over the hydrograph duration, the second parameter describes the effect of overall hydrograph volume, and the third is a time ratio to account for the relative duration of the rising and receding hydrograph limbs (i.e. hydrograph shape). Graf and Suszka (1985) used the change in water surface elevation, $\Delta H$, to define hydrograph flashiness through the derivation of an unsteadiness parameter, i.e.

$$
\Gamma_{H G}=\frac{1}{u_{* 0}} \frac{\Delta H}{T_{h}}
$$

This unsteadiness parameter is, thus, equivalent to the first non-dimensional group in brackets in Eq. (5) when $h$ is substituted by $\Delta H$ [i.e. representing the difference in water depths $\left(h_{p}-\right.$ $h_{0}$ ) between the peak, $Q_{\text {peak }}$, and base, $Q_{0}$, flows, respectively], and $u *=u_{*}$ [i.e. where $u *_{0}$ is the bed shear velocity under base (antecedent) flow conditions].

Similarly, a total water work, $W_{k}$, parameter has been previously proposed (Yen and Lee, 1995) to represent the influence of the total water volume discharged during individual hydrograph events on bed-load sediment transport, i.e. 


$$
W_{k}=\frac{u_{* 0}^{2} V_{h}}{g h_{0}^{3} B}
$$

Again, this parameter is equivalent to the second non-dimensional group in Eq. (3), when flow depth, $h=h_{0}$, and shear velocity, $u *=u * 0$, [i.e. for base (antecedent) flow conditions]. The water work parameter, $W_{k}$, was subsequently adopted by Lee et al. (2004) and Bombar et al. (2011) to describe the influence of the total discharged water volume (of unsteady flows) on bed-load sediment yields for idealized triangular and trapezoidal hydrographs. It is worth noting at this stage that $\Gamma_{H G}$ and $W_{k}$ are independent variables as different hydrographs with the same $\Gamma_{H G}$ value (i.e. fixed $\Delta H / T_{h}$ ratios) may have different $W_{k}$ values (i.e. variable $V_{h}$ values) and vice versa. Hence, it is possible to rewrite the general functional relationship of Eq. (5) as follows:

$W_{t}^{*}=f\left(\Gamma_{H G}, W_{k}, \eta\right)$

where $\eta=T_{r} / T_{f}$ is defined as a hydrograph asymmetry parameter. Hence, when $\eta=1.0$, the hydrograph is symmetrical in shape (i.e. $T_{r}=T_{f}$ ), while $\eta>1$ and $\eta<1$ represent unsymmetrical (skewed) hydrographs with relatively longer and shorter rising limb durations (i.e. longer and shorter times to peak flow), respectively. The calculated unsteadiness, $\Gamma_{H G}$, total water work, $W_{k}$, and asymmetry, $\eta$, parameter ranges for all design hydrographs tested are listed in Table 2.

\section{Experimental Results}

\subsection{Temporal Variation in Bed Surface Properties}

As indicated previously, bed surface layer composition was measured during the unsteady flow hydrographs using the Sedimetrics bed imaging technique. Temporal changes to the median grain size, $d_{s 50}$, of the bed surface and its fractional composition, $f_{i}$, could, thus, be assessed throughout each run. In this regard, Figures 7(a) and (b) show the variation in $d_{s 50}$ 
sizes for the uni-modal (UM) and bimodal (BM) sediment mixtures, respectively, over the duration of the HY1_1a - 1c hydrographs (Figure 6 and Table 2). Corresponding values of the $d_{b 50}$, obtained from the bed-load samples collected in the sediment trap, are also shown for comparison purposes. For all experimental runs, the initial $d_{s 50}$ measurements were significantly larger than the median grain size $\left(d_{50}=5.0 \mathrm{~mm}\right)$ for both $\mathrm{UM}$ and BM design mixtures. This indicates that sediment placement within the flume bed and/or sub-threshold, antecedent flow conditions led to a general coarsening of the bed surface layer prior to the onset of bed-load transport under the specific flow hydrographs. Similar findings were also obtained from the experimental study of Mao et al. (2010), where image-derived bed surface median grain sizes were generally coarser than $d_{50}$ sizes for the original sediment mixtures tested. The mechanisms for this initial bed surface coarsening clearly depend on bed grading and, specifically, the ability of finer fractions at the surface to progressively migrate into deeper subsurface pore space within the gravel mixture (e.g., Wilcock et al., 2001; Wilcock and Crowe, 2003), leading to the formation of a coarsened or armored surface layer (e.g., Wilcock and DeTemple, 2005).

After this initial bed surface coarsening, subsequent variations in $d_{s 50}$ values due to sediment transport during the hydrograph flow are shown to either reduce monotonically, indicating bed surface fining (Figures 7a-1, b-1, and b-2), or remain relatively unchanged (Figures 7a-2, a-3, and b-3). It appears that this surface fining effect is most apparent for both UM and BM mixtures under hydrographs with shorter rising limbs (i.e. $\eta<1$, Figures $7 \mathrm{a}-1$, b-1), compared to those with longer rising limbs (i.e. $\eta>1$, Figures $7 \mathrm{a}-3$, b-3), where overall fining or coarsening of the bed surface is marginal over the duration of the hydrograph. This variability in median bed surface grain size, $d_{s 50}$, is also reflected by temporal changes in the measured proportionality coefficients, $f_{i}$, for the fine, medium, and coarse size classes present 
in the bed surface layer (Figure 8). In general, for runs with overall reductions in $d_{s 50}$ values (i.e. surface fining), $f_{i}$ values for the coarse and fine classes were shown typically to reduce and increase, respectively, over the same duration (see Figure 8a-1, 8b-1, and 8b-2). For the other runs showing little variation in $d_{s 50}$ values, $f_{i}$ values also tended remain relatively constant.

It is informative to compare the $d_{s 50}$ values measured in the bed surface layer with corresponding $d_{b 50}$ values obtained from sediment trap bed-load samples. As expected, $d_{b 50}$ was generally lower than $d_{s 50}$ throughout the hydrograph duration in all tests, although no clear relationship was demonstrated between these median values in any run. In comparison to the relatively minor changes observed in the $d_{s 50}$ values, corresponding distributions of $d_{b 50}$ values showed strong variability over the hydrographs, with distinctive periods of increasing and decreasing $d_{b 50}$ during the rising and receding limbs, respectively.

\subsection{Temporal Variation in Bed-load Transport}

Figure 9 presents temporal variations in the normalized bed-load transport rates, $q_{b}{ }^{*}$, for the predefined fine, medium, and coarse fractions of the UM and BM sediment mixtures (see Table 1) over the duration of the hydrographs with different $\eta$ values (i.e. HY1_1a - c, Figure 6a and Table 2). For the UM sediment mixture (Figure 9a), the results indicated that total transport rates, $q_{b}{ }^{*}$, typically reached their peak value after peak flow conditions had been attained in the flume (i.e. during the receding hydrograph limb). In relation to fractional transport rates, the coarse and medium size classes were found to typically attain their peak $q_{b i}{ }^{*}$ values either on the rising hydrograph limb or around peak flow conditions, while peak $q_{b i}{ }^{*}$ values for the fine size class were typically attained during the receding limb. This fraction-dependent temporal lag is clearly shown in the symmetrical hydrograph run $(\eta=1$, 
Figure 9a-1), where peak $q_{b i}{ }^{*}$ values for the coarse, medium, and fine size classes were attained at $t^{*}=34918,42480$ and 50041, respectively (with the peak flow rate $Q^{*}$ occurring at $\left.t^{*}=45368\right)$. Similar total and fractional temporal lag effects were observed for the BM sediment runs (Figure 9b), with peak $q_{b i}{ }^{*}$ values for the coarse and fine size classes again typically attained on the rising and receding limbs. Indeed, for the range of flow hydrographs (and sediment mixtures) tested, the normalized temporal lag $\Delta t^{*}$ between peak $q_{b i}{ }^{*}$ values for the coarse and fine size classes was either $\Delta t^{*}=7562$ or 15124 , based on the 10 minute bedload sampling frequency employed in all runs.

Comparing the magnitude of $q_{b}{ }^{*}$ and $q_{b i}{ }^{*}$ values attained for the UM and BM sediments (Figures 9a and b, respectively), it is interesting to note that peak values of the total transport rate, $q_{b}{ }^{*}$,were typically higher for BM sediment compared to UM sediment runs. Similarly, larger peak $q_{b i}{ }^{*}$ values were also typically obtained for the coarse and fine size classes within the BM sediment runs, whilst corresponding peak $q_{b i}{ }^{*}$ values for the medium size class are broadly equivalent in both UM and BM runs.

The influence of hydrograph unsteadiness, $\Gamma_{H G}$, and total water work, $W_{k}$, on $q_{b}{ }^{*}$ and $q_{b i}{ }^{*}$ values is shown in Figure 10 for runs conducted with BM sediment under symmetrical hydrographs of varying magnitude (i.e. HY1_2a-d, Figure 6b and Table 2). As with Figure 9 , peak values of the total transport rate, $q_{b}{ }^{*}$, tended to occur consistently on the receding hydrograph limb, indicating a pronounced lag between peak flow rates and bed-load transport rates. In addition, the observed temporal lag between peak fractional, $q_{b i}{ }^{*}$, values for the coarse and fine size classes was again observed to be $\Delta t^{*}=7562$ or 15124 (i.e. one or two sampling periods). It is also apparent from Figure 10 that measured total and fractional bedload transport rates generally reduced with peak flow rate, $Q_{\text {peak }}$ (i.e. reducing $\Gamma_{H G}$ and $W_{k}$ values, Figure $10 \mathrm{a} \rightarrow \mathrm{d}$ ). In addition, measured $q_{b i}{ }^{*}$ values for the fine sediment class were 
typically an order of magnitude higher than corresponding $q_{b i}{ }^{*}$ values for both the medium and coarse size classes, in accord with the BM sediment runs conducted under hydrographs with varying $\eta$ (Figure 9b).

Clearly, some level of uncertainty is associated with the specific values of $q_{b}{ }^{*}$ and $q_{b i}{ }^{*}$ presented in Figures $8-10$ due, for example, to averaging processes associated with the trap sampling procedure (e.g., trap width and sampling time). Analysis and evaluation of this uncertainty would require a statistically-significant number of repeat runs to be conducted for each individual experimental condition, which is beyond the scope of the current study.

\subsection{Fractional Bed-load Mobility}

It has previously been shown in graded sediment beds that hiding effects for smaller particles and the protrusion of coarser grains have significant influence on the magnitude of fractional bed-load transport, and the temporal lag effects between different fractions, when compared to corresponding transport rates for uniform or quasi-uniform sediments (e.g. Einstein, 1950; Parker and Klingeman, 1982; Komar, 1987a, 1987b; Wilcock, 1993; Wu et al., 2000; Shvidchenko et al., 2001). It is, thus, important to establish how fractional mobility for the UM and BM sediment mixtures tested here may influence observed bed-load transport rates over the hydrograph flows considered. In this regard, the fractional bed-load mobility hypothesis developed by Parker and Klingeman (1982) can be adopted here, as follows: $\psi_{i}=\frac{p_{i}}{F_{i}}$

where $p_{i}$ and $F_{i}$ represent the fractional proportions (by weight) in the collected bed-load sample and original design sediment mixture, respectively. This fractional mobility parameter, $\psi_{i}$, can, thus, be interpreted physically, as follows: (i) $\psi_{i}=1$, corresponding to 
equal mobility for a particular grain size (i.e. equivalent proportions are present in the transported bed-load and graded sediment bed mixture); (ii) $\psi_{i}>1$, representing enhanced transport mobility when compared to the proportion contained in the sediment bed mixture, possibly due to surface grain protrusion effects; and (iii) $\psi_{i}<1$, representing reduced transport mobility compared to that anticipated from the proportion contained in sediment bed mixture, possibly due to grain hiding effects.

Figure 11 presents the temporal variation in the fractional bed-load mobility parameter, $\psi_{i}$, for the UM (Figure 11a) and BM (Figure 11b) sediments under design hydrographs with variable $\eta$ (i.e. HY1_1a - c, Figure 6a and Table 2). Although there is considerable scatter in the plotted data, a general trend was observed for the coarse size class in both UM and BM sediment mixtures whereby $\psi_{i}$ values typically increased during the rising hydrograph limb before reducing significantly during the receding limb. The main difference in the fractional mobility of the coarse fraction within each sediment mixture was the relative magnitude of $\psi_{i}$ values attained over the hydrographs, with $\psi_{i}$ values up to 1.4 (i.e. enhanced mobility, Figure 11a-2) observed for the UM sediment during the rising limb and peak flow regions. By contrast for the BM sediment, corresponding values of $\psi_{i} \leq 1$ were obtained throughout the hydrographs, indicating generally reduced mobility. Corresponding fractional mobility, $\psi_{i}$, values for the finer size class also indicted distinct trends for both UM and BM sediments. Values of $\psi_{i}<1$ (i.e. reduced mobility) were typically attained during the rising hydrograph limbs, with $\psi_{i}$ values also often shown to reduce as flow rate increased. Towards the end of the rising limb and/or around peak flow, $\psi_{i}$ values for both sediment mixtures began to increase, initially towards equal mobility conditions (i.e. $\psi_{i}=1$ ), and subsequently to $\psi_{i}>1$ values (up to 2.1, see Figures 11a-1, a-3, and b-2) during the latter stages of the receding 
hydrograph limb, indicating significantly enhanced mobility in this region. In contrast to these strongly-defined (and largely opposite) fractional mobility trends for the coarse and fine size classes, $\psi_{i}$ values for the medium size class were shown to remain relatively consistent over the hydrograph duration. These values typically ranged between $\psi_{i}=1.0-1.5$ and $1.0-$ 1.7 for the UM and BM sediments, respectively, hence indicating generally enhanced mobility (i.e. $\psi_{i}>1$ ). Similar plots of fractional mobility for the design hydrographs with varying $\Gamma_{H G}$ and $W_{k}\left(\mathrm{HY} 1 \_2 \mathrm{a}-\mathrm{c}\right.$, Figure $6 \mathrm{~b}$ and Table 2$)$ were also found to largely reflect these magnitudes and variabilities in $\psi_{i}$ values for the different size classes.

The foregoing observations on fractional mobility are confirmed by the variations in $d_{b 50}$ values for bed-load samples measured throughout the HY1_1 hydrograph durations (see Figure 7). As these distributions consistently show increasing $d_{b 50}$ values during the rising limb and decreasing $d_{b 50}$ values during the receding limb, it is apparent that the mobility of larger grain sizes increases during the rising limb, while finer grain sizes become increasingly mobile during the receding limb, when the transport rate of larger size fractions reduces.

\section{Discussion}

The two graded sediment mixtures tested in the present study have been shown to demonstrate both analogous and divergent trends in their observed bed-load transport characteristics over the range of unsteady flow hydrographs tested. These specific trends were associated primarily with temporal variations in (i) bed surface properties (i.e. median surface grain size, $d_{s 50}$, and fractional composition, $f_{i}$ ), (ii) total, $q_{b}{ }^{*}$, and fractional, $q_{b i}{ }^{*}$, bedload transport rates, and (iii) fractional bed-load mobility, $\psi_{i}$; as well as temporal lag effects between the unsteady flow hydrograph conditions and (total and fractional) sediment transport rates. Each of these issues is discussed in greater depth here. 


\subsection{Influence of Sediment Composition on Transport Behavior}

In order to investigate further the influence of sediment mixture composition on the observed fractional transport behavior, a relative transport yield parameter, $\chi_{i}$, can be defined to compare directly fractional bed-load yields, $W_{t i}$, (over the hydrograph duration) for the UM and $\mathrm{BM}$ sediment beds (i.e. $W_{t i, U M}$ and $\left.W_{t i, B M}\right)$, i.e.

$\chi_{i}=\frac{W_{t i, B M} F_{i, U M}}{W_{t i, U M} F_{i, B M}}$

where $F_{i, U M}$ and $F_{i, B M}$ represent the original proportion of fraction $i$ in the $\mathrm{UM}$ and BM mixtures, respectively. This transport yield parameter, $\chi_{i}$, therefore, compares the relative ease by which a particular fraction or size class is transported in each mixture, in relation to its fractional representation in the mixtures. In this regard, Figure $12 \mathrm{a}$ shows that $\chi_{i}$ values for the fine, medium, and coarse size classes consistently exceed unity (with $\chi_{i}$ up to 3.6) for the HY1_1 hydrograph runs (i.e. Figure 6a and Table 2). This indicates clearly that fractional transport yields from the BM sediment mixture are significantly in excess of corresponding yields obtained from the UM mixture under equivalent hydrograph flow conditions, implying that the UM sediment bed is inherently more stable than the BM bed. This was also inferred previously from the increased total, $q_{b}{ }^{*}$, and fractional, $q_{b i}{ }^{*}$, transport rates observed for BM sediment, compared to UM sediment (see Figure 9). The main difference between the two graded sediment mixtures was the depleted proportion of $d_{i}=5.15 \mathrm{~mm}$ and $7.15 \mathrm{~mm}$ size fractions in the BM mix, which were replaced by additional finer $\left(d_{i}=3.4 \mathrm{~mm}\right)$ and coarser $\left(d_{i}=9.0 \mathrm{~mm}\right)$ grades (see Figure 2$)$. Hence, the fact that proportionately less sediment from the medium size class is transported from the UM graded bed compared to from the BM bed (i.e. $\chi_{i}=1.92-2.38$, with $F_{i, U M} / F_{i, B M}=1.86$ ) suggests that this specific material size acts to stabilize the UM sediment bed. 
This stabilization effect can be examined by the application of a reference bed-load transport threshold to describe bed-load motion (i.e. Shvidchenko and Pender, 2000). If this threshold level is set, for example, at $q_{b}{ }^{*}=10^{-4}$, it can be demonstrated from Figure 9 that the corresponding $Q^{*}$ values at which this transport threshold is reached are typically lower $\left(Q^{*} \approx\right.$ $1.12-1.24)$ for the BM sediment compared to the UM sediment $\left(Q^{*} \approx 1.34-1.45\right)$. This suggests that higher normalized flow rates (i.e. corresponding to higher shear stress conditions) are required to attain this transport threshold for the UM sediment, again indicating more stable bed conditions. Similar fractional bed-load transport, $q_{b i}{ }^{*}$, thresholds could also be applied directly to the fine, medium, and coarse size classes to again demonstrate the generally lower $Q^{*}$ values required within the BM sediment runs to attain these transport thresholds.

It is interesting to investigate the possibility that these bed stabilization effects may also be linked to absolute grain-size-related processes that can affect fractional sediment entrainment properties. Shvidchenko and Pender (2000) revealed a dependence on absolute grain size of the flow conditions required to reach a specific sediment transport intensity threshold for different sized uniform sediment beds. They found that relatively higher flow conditions (i.e. shear stresses) were required for $d=5 \mathrm{~mm}$ particles, compared to both finer and coarser grains. In another study, Saadi (2008) ran antecedent (steady) flow conditions to observe the stability development of a bimodal sediment mixture, with $d=4 \mathrm{~mm}$ particles shown to be intrinsically more stable than other grain sizes, based on the analysis of critical shear stresses. Within the current study, these absolute grain-size-related effects can be investigated by comparing $\chi_{i}$ values for individual size fraction constituents of the UM and BM sediment mixtures, tested under the equivalent steady (ES) flow conditions (Table 2). Figure 12b 
shows that $\chi_{i} \geq 1$ (up to 6.8 ) typically across the range size fractions, with $\chi_{i}<1$ observed only for the coarsest fraction $\left(d_{i}=14.6 \mathrm{~mm}\right)$. However, for the $d_{i}=5.15$ and $7.15 \mathrm{~mm}$ fractions significantly depleted in the BM sediment bed (i.e. $F_{i, U M} / F_{i, B M} \approx 1.92$ and 1.80 ), $\chi_{i} \approx$ 1.4 and 2.3, respectively, indicating that the fractional transport yields from BM sediment bed were about $73 \%$ and $130 \%$ of the equivalent fractional yields from the UM bed. This again highlights the stabilizing influence of these medium-sized fractions in the UM sediment bed.

Physical explanations for these stabilization and absolute grain size effects remain unclear, but may speculatively be related to (i) the specific nature of flow-particle interactions that result in the entrainment of different sized grains from a graded bed surface, and/or (ii) differences in the packing arrangements between bimodal and uni-modal beds that make the former more susceptible to erosion.

\subsection{Influence of Hydrograph Characteristics on Transport Behavior}

Results from the study have indicated a strong influence from the hydrograph flow conditions (defined by $\Gamma_{H G}, \eta$, and $W_{k}$ ) on (i) bed-load composition and mobility (i.e. $d_{b 50}$ and $\psi_{i}$ ), and

(ii) total, $q_{b}{ }^{*}$, and fractional, $q_{b i}{ }^{*}$, bed-load transport rates. Many of these sediment transport related parameters were also shown to vary significantly between the rising and receding limbs of the hydrograph. In particular, while bed surface composition (i.e. $d_{s 50}$ and $f_{i}$, Figures 7 and 8) tended to become finer or remain consistent over the hydrograph duration, bed-load composition (i.e. $d_{b 50}$, Figure 7) typically coarsened over the rising limb and became finer over the receding limb. This generally corresponded to the increased mobility of coarser size classes during the rising limb and increased finer size class mobility during the receding limb. The reason that these distinct compositional changes in bed-load are not reflected in the observed changes to bed surface composition may be two-fold. Firstly, the sample bed area 
$(0.2 \times 0.15 \mathrm{~m})$ on which the surface layer composition was calculated using the Sedimetrics image analysis technique was, by necessity, relatively small. Hence, if the bed-load composition entering this area is largely similar to the composition leaving it, then strong changes in the bed surface layer composition may not be observed. Secondly, although calibration of the Sedimetrics bed imaging technique against directly collected bed samples was reasonable for both UM and BM mixtures (Figure 5), there were significant doubts over the accuracy of this visualization technique under conditions of general bed motion.

The specific influence of flow conditions during the rising and receding limbs on both the total and fractional bed-load transport rates can also be further investigated through definition of relative transport yield ratios, i.e.

$$
\begin{gathered}
\zeta=\frac{W_{t, r}}{W_{t, f}} \\
\zeta_{i}=\frac{W_{t i, r}}{W_{t i, f}}
\end{gathered}
$$

where subscripts $r$ and $f$ refer to the transport yields measured over the duration of the rising and receding limbs, respectively. Figure 13 shows total, $\zeta$, and fractional, $\zeta_{i}$, yield ratios plotted against the hydrograph flow parameters $\eta$ and $\Gamma_{H G}$. It is apparent from Figures $13 \mathrm{a}-\mathrm{c}$ that $\zeta$ and $\zeta_{i}$ values increase monotonically with increasing $\eta$, indicating that relatively longer duration rising limbs (i.e. $\eta>1$ ) result in larger proportions of the UM and BM bed-load yields being transported during the rising limbs. More generally, the results suggest that overall proportions of bed-load yields transported during either the rising or receding limbs depend largely on their respective durations.

Comparing $\zeta$ and $\zeta_{i}$ values for the UM and BM sediment mixtures, it is also clear that the 
UM sediment bed was relatively more active during the rising limb (i.e. higher $\zeta$ and $\zeta_{i}$ values) compared to the BM bed. It is suggested here that this may have been due partly to the larger fractional proportion of fine size class sediment in the BM mixture $\left(F_{i} \sim 0.39\right)$ than in the UM mixture $\left(F_{i} \sim 0.30\right)$, which was typically shown to have increased mobility $\psi_{i}$ during the receding hydrograph limb (Figure 11). This may also be reflected, to some extent, by the runs in which bed surface composition was shown to become finer over the hydrograph duration (albeit, this was not a universal effect). Overall, however, it is anticipated that these temporally-varying, bed-load mobility effects will be strongly associated with changes in bed composition and structure (i.e. fractional availability, grain hiding, and protrusion effects) prior to and during the hydrograph events. This topic, in particular, therefore, warrants further investigation.

Additionally, the influence of hydrograph unsteadiness, $\Gamma_{H G}$, on the total, $\zeta$, and fractional, $\zeta_{i}$, yield ratios is shown in Figure 13d. This plot again shows a monotonic increase in both $\zeta$ and $\zeta_{i}$ values for BM sediment runs as $\Gamma_{H G}$ increases, suggesting that flashier hydrographs (i.e. shorter duration events with increased peak flows) result in increasing proportions of the overall sediment yield being transported on the rising limb. However, it is noted from Figure $13 \mathrm{~d}$ that the vast majority of $\zeta$ and $\zeta_{i}$ values lie below unity, with larger sediment yields, thus, obtained during the receding flow conditions. This may suggest that hydrographs of higher unsteadiness are likely to initiate general bed-load transport earlier in the unsteady flow event under lower flow (and shear stress) conditions. Such a hypothesis appears to be reflected by the observed magnitude and variability of transport rates, $q_{b}{ }^{*}$, for the BM sediment runs over the rising limbs of the HY1_2 hydrographs (Figure 10). These plots indicate that a prescribed transport rate, $q_{b}{ }^{*}$, threshold can be attained under lower $Q^{*}$ flows when $\Gamma_{H G}$ is increased. These findings are generally concur with the study of Kuhnle (1992) based on sediment 
transport measurements in two small natural streams. Here, bed-load transport rates were typically found to be larger during the rising limb of high magnitude hydrograph events, while for lower magnitude hydrograph events, one stream demonstrated the opposite trend.

Finally, Figures $13 \mathrm{~b}-\mathrm{d}$ also indicated strongly that, for any given hydrograph asymmetry, $\eta$, or unsteadiness, $\Gamma_{H G}, \zeta_{i}$ values measured for the coarse size class consistently exceeded corresponding $\zeta_{i}$ values obtained for the medium size class, which in turn were greater than the $\zeta_{i}$ values for the fine size class. This suggests that while the coarser particles are more often preferentially transported during the rising hydrograph limb than the receding limb (i.e. $\zeta_{i}>1$ ), the fine size class is more often preferentially transported during the receding limb (i.e. $\left.\zeta_{i}<1\right)$. When combined with the previously observed variability in temporal lag effects for these different size classes, this clearly reveals a strong size-dependency in the fractional response of graded sediment beds, and resulting bed-load transport, to unsteady flow hydrographs.

\subsection{Comparisons with Equivalent Steady (ES) Flow}

The relative influence of hydrograph flow parameters $\left(\eta, \Gamma_{H G}\right.$, and $\left.W_{k}\right)$ on the overall UM and BM sediment yields obtained over the hydrograph durations are shown in Figures 14a and $b$. It is apparent from these plots that the influence of shape $\eta$ (Figure 14a) is significantly lower than both $\Gamma_{H G}$ and $W_{k}$, where sediment yields increase significantly as both $\Gamma_{H G}$ and $W_{k}$ increase. (Figure 14b, BM only). It should be re-iterated at this stage that the magnitude and unsteadiness of hydrograph events will be independent of each other in nature and, thus, the influence of both hydrograph unsteadiness, $\Gamma_{H G}$, and total water work, $W_{k}$, on sediment transport should be considered independently. However, it is also acknowledged that the limitation of the parametric conditions considered in this study meant that the 
independent influence of $\Gamma_{H G}$ and $W_{k}$ on sediment yield, $W_{t}^{*}$, was not directly assessed. It is hypothesized here that $W_{t}^{*}$ values would be expected to increase with independent increases in $\Gamma_{H G}$ or $W_{k}$. Clearly larger magnitude hydrograph events (i.e. higher water work, $W_{k}$, for fixed unsteadiness, $\Gamma_{H G}$ ) or events that are flashier in nature (i.e. higher unsteadiness, $\Gamma_{H G}$, for fixed water work, $W_{k}$ ), would be expected to transport more sediment than lower magnitude or less unsteady hydrograph events. In this sense, comparing the total bed-load transport yields obtained for the UM and BM sediments over the HY1_1 hydrograph conditions with the corresponding yields obtained under equivalent, steady (ES) flow conditions (see Table 2) reveals the limit of the effect of flow unsteadiness, $\Gamma_{H G}$, on bed-load transport. When considered over the same duration as the HY1_1 hydrographs (i.e. equivalent $W_{k}$, Table 2), Figure $14 \mathrm{c}$ shows clearly that the total sediment yields obtained under the ES flow condition are substantially lower (up to an order of magnitude) for both UM and BM mixtures than under the HY1_1 hydrographs, in agreement with the foregoing hypothesis.

The findings from the current study also serve to highlight the significant difficulties experienced in predicting sediment transport rates and/or yields within natural streams and rivers that are subject to a wide range of unsteady flow hydrograph events of different magnitudes and unsteadiness. Further progress in this area, therefore, requires (i) improved understanding of the temporal response of gravel beds at field scales to specific types or combinations of flood events, and (ii) the inclusion of a wider range of unsteady flow parameters within graded sediment transport models tasked with providing predictions of these transport rates and yields. In this sense, many of the non-dimensional flow and transport parameters utilized in the current study provide a useful starting point for the assessment of interactions between unsteady flow hydrographs and temporally-varying bed surface properties and sediment transport processes at field prototype scales. 


\section{Conclusions}

Two different graded sediment mixtures were tested to investigate the influence of unsteady flow hydrographs on the temporal variations in bed-load sediment transport rates, bed surface composition, and relative fractional mobility of the different size classes contained within the mixtures. Analysis of total and fractional transport rates from bed-load samples collected throughout the hydrographs revealed that the coarser size class $(d>8.0 \mathrm{~mm})$ had increased mobility during the rising hydrograph limb and tended to attain the peak fractional transport rate before (or close to) peak flow conditions being reached. By contrast, the fine size class ( $d$ $<4.0 \mathrm{~mm}$ ) tended to have increased mobility during the receding limb and typically attained its peak transport rate subsequent to the peak flow. Corresponding temporal variations in (i) the median grain size, $d_{b 50}$, for the transported bed-load, and (ii) the fractional mobility, $\psi_{i}$, parameter, also demonstrated that the bed-load typically coarsened over the rising limb, before becoming finer again during the receding limb. By contrast, the bed surface composition was found either to become slightly finer over the duration of the hydrograph or remain essentially unchanged.

The influence of hydrograph characteristics on these sediment transport and bed properties was investigated by varying defined, non-dimensional shape (asymmetry), $\eta$, unsteadiness, $\Gamma_{H G}$, and total water work, $W_{k}$, parameters. These were shown to have a strong influence on the relative total and fractional transport yields that were attained during the rising and receding hydrograph limbs. Results indicated that increased transport yields were typically obtained during the rising limb when hydrograph magnitude, $W_{k}$, and/or unsteadiness, $\Gamma_{H G}$, were increased. It was suggested that this may result from specific transport, $q_{b}{ }^{*}$, threshold rates being reached earlier and under lower flow conditions for hydrograph events with 
higher $W_{k}$ and $\Gamma_{H G}$ values, compared to lower magnitude, less unsteady hydrographs.

Experimental results also indicated that the bimodal (BM) sediment mixture was less stable than the uni-modal (UM) mixture under equivalent hydrograph flow conditions, resulting in higher overall transport rates and yields. In this respect, the medium size class $(d=4-8 \mathrm{~mm})$ was found to be crucial to bed stabilization; hence, the relative depletion of this size class in the BM sediment bed $\left(F_{i}=0.22\right)$, compared to its relative abundance in the UM sediment bed $\left(F_{i}=0.41\right)$, was thought to be significant to the increased transport rates and yields observed.

The findings from this study have significant benefits for improved understanding of sediment transport processes arising from natural stream and river beds with different gravel size distributions and grading characteristics (i.e. uni-modal or bimodal) that are subjected to a wide range of unsteady flow conditions. In particular, enhanced knowledge of the specific parametric dependences of unsteady flow hydrograph events on graded sediment transport and bed properties is clearly essential to improve predictive capabilities of the resulting temporal variations in bed surface composition, fractional mobility, and sediment transport rates and yields.

\section{Acknowledgements}

Financial support for Le Wang was provided by the UK Overseas Research Student Awards Scheme (ORSAS) and a James Watt Scholarship tenable in the Institute of Infrastructure and Environment at Heriot-Watt University, Edinburgh, UK. The authors are also grateful for the comments provided by two anonymous reviewers that have led to significant improvements in the paper. 


\section{References}

Bell, R. G. 1980. Non-Equilibrium Bed load Transport by Steady and Non-Steady Flows, Report No. 80/23, Department of Civil Engineering, University of Canterbury, New Zealand.

Berta, A. M. and Bianco, G. 2010. An expression for the water-sediment moving layer in unsteady flows valid for open channels and embankments. Natural Hazards and Earth System Sciences, Vol. 10, pp.1051-1059.

Bombar, G., Elçi, Ş., Tayfur, G., Güney, M. Ş. and Bor, A. 2011. Experimental and numerical investigation of bed-load transport under unsteady flows. Journal of Hydraulic Engineering, Vol. 137, No. 10, pp.1276-1282.

Einstein, H. A. 1950. The bed-load function for sediment transportation in open channel flows. US Department of Agriculture, Soil Conservation Service, Technical Bulletin No. 1026.

Frey, P., Ducottet, C. and Jay, J. 2003. Fluctuations of bed load solid discharge and grain size distribution on steep slopes with image analysis. Experiments in Fluids, Vol. 35, No. 6, pp.589-597.

Ghimire, B. and Deng, Z. Q. 2012. Event flow hydrograph-based method for modeling sediment transport. Journal of Hydrologic Engineering, Vol. 18, No. 8, pp. 919-928.

Graf W. H. and Suszka, L. 1985. Unsteady flow and its effect on sediment transport. Proceedings of the 21st IAHR Congress, Melbourne, Australia, pp. 1-5.

Graf, W. H. and Qu, Z. 2004. Flood hydrographs in open channels. Proceedings of the ICEWater Management, Vol. 157, No. 1, pp.45-52.

Graham, D.J., Reid, I. and Rice, S.P. 2005. Automated sizing of coarse grained sediments: Image-processing procedures. Mathematical Geology, Vol. 37, No. 1, pp.1-28.

Griffiths, G. A. 1976. Transport of Bed load by Translation Waves in an Alluvial Channel, 
Report No. 76-1, Department of Civil Engineering, University of Canterbury, New Zealand.

Komar, P. D. 1987a. Selective grain entrainment by a current from a bed of mixed sizes: A reanalysis. Journal of Sedimentary Research, Vol. 57, No. 2, pp.203-211.

Komar, P. D. 1987b. Selective gravel entrainment and the empirical evaluation of flow competence. Sedimentology, Vol. 34, No. 6, pp.1165-1176.

Kuhnle, R. A. 1992. Bed load transport during rising and falling stages on two small streams. Earth Surface Processes and Landforms, Vol. 17, No. 2, pp.191-197.

Lee, K. T., Liu, Y. L. and Cheng, K. H. 2004. Experimental investigation of bed-load transport processes under unsteady flow conditions. Hydrological Processes, Vol. 18, No. 13, pp.2439-2454.

Mao, L., Cooper, J. and Frostick, L. 2010. Hydrograph effects on sediment transport and surface grain sorting and organization, Gravel-bed Rivers 7 Conference, Tadoussac, Québec, 6-10 September 2010 (poster).

Mao, L. 2012. The effect of hydrographs on bed load transport and bed sediment spatial arrangement, Journal of Geophysical Research, Vol. 117, F03024, doi: 10.1029/2012JF002428.

Parker, G. 1990. Surface-based bedload transport relation for gravel rivers. Journal of Hydraulic Research, Vol. 28, No. 4, pp.417-436.

Parker, G. and Klingeman, P. C. 1982. On why gravel bed streams are paved. Water Resources Research, Vol. 18, No. 5, pp.1409-1423.

Parker, G., Hassan, M. and Wilcock, P. 2007. Adjustment of the bed surface size distribution of gravel-bed rivers in response to cycled hydrographs. Developments in Earth Surface Processes, Vol. 11, pp.241-285.

Phillips, B. C. and Sutherland, A. J. 1990. Temporal lag effect in bed load sediment transport. 
Journal of Hydraulic Research, Vol. 28, No. 1, pp.5-23.

Piedra, M. M. 2010. Flume investigation of the effects of sub-threshold rising flows on the entrainment of gravel beds, Doctoral Thesis, University of Glasgow, UK.

Saadi, Y. 2008. Fractional critical shear stress at incipient motion in bimodal sediment. Civil Engineering Dimension, Vol. 10, No. 2, pp.89-98.

Shvidchenko, A. B. and Pender, G. 2000. Flume study of the effect of relative depth on the incipient motion of coarse uniform sediments. Water Resources Research, Vol. 36, No. 2, pp.619-628.

Shvidchenko, A. B., Pender, G. and Hoey, T. B. 2001. Critical shear stress for incipient motion of sand/gravel streambeds. Water Resources Research, Vol. 37, No. 8, pp.22732283.

Wilcock, P. R. 1993. Critical shear stress of natural sediments. Journal of Hydraulic Engineering, Vol. 119, No. 4, pp.491-505.

Wilcock, P. R., Kenworthy, S. T. and Crowe, J. C. 2001. Experimental study of the transport of mixed sand and gravel. Water Resources Research, Vol. 37, No. 12, pp.3349-3358.

Wilcock, P. R. and Crowe, J. C. 2003. Surface-based transport model for mixed-size sediment. Journal of Hydraulic Engineering, Vol. 129, No. 2, pp.120-128.

Wilcock, P. R. and DeTemple, B. T. 2005. Persistence of armor layers in gravel-bed streams. Geophysical Research Letters, Vol. 32, L08402, doi: 10.1029/2004GL021772.

Wu, W., Wang, S. S. and Jia, Y. 2000. Nonuniform sediment transport in alluvial rivers. Journal of Hydraulic Research, Vol. 38, No. 6, pp.427-434.

Yen, C. L. and Lee, K. T. 1995. Bed topography and sediment sorting in channel bend with unsteady flow. Journal of Hydraulic Engineering, Vol. 121, No. 8, pp.591-599.

\section{Figure Captions}


Figure 1: Schematic representation of experimental flume set-up.

Figure 2: Grain size distributions for (a) uni-modal (UM) and (b) bimodal (BM) graded sediment mixtures.

Figure 3: (a) Photograph showing the bed-load sediment trap positioned beneath the flume, and (b) schematic diagram of the bed-load sediment trap arrangement.

Figure 4: (a) High-resolution image of the in-situ bed surface layer (BM sediment), and (b) processed image by Sedimetrics for determination of grain size distribution on the bed surface.

Figure 5: Comparison of grain size distributions from the Sedimetrics image analysis technique and direct bed sampling.

Figure 6: Design hydrographs used in study (a) HY1_1 (variable asymmetry, $\eta$, constant unstreadiness, $\Gamma_{H G}$, and total water work, $W_{k}$, Table 2); and (b) HY1_2 (constant $\eta$, variable $\Gamma_{H G}$ and $W_{k}$, Table 2).

Figure 7: Temporal variation in median grain sizes of the bed surface layer $d_{s 50}$ and bed-load transport $d_{b 50}$ for (a) UM sediment runs, and (b) BM sediment runs with HY1_1 hydrograph conditions (see Table 2). Hydrograph flow $Q$ profile shown for comparative purposes.

Figure 8: Temporal variation in the proportionality coefficients, $f_{i}$, of fine, medium, and coarse size classes present in the bed surface layer for (a) UM sediment runs, and (b) BM sediment runs with HY1_1 hydrograph conditions (see Table 2). Normalized hydrograph flow, $Q^{*}$, distribution shown for comparative purposes.

Figure 9: Temporal variation in normalized total $q_{b}{ }^{*}$ and fractional $q_{b i}{ }^{*}$ bed-load transport rates for (a) UM sediment runs and (b) BM sediment runs with HY1_1 hydrograph 
conditions (see Table 2). Normalized hydrograph flow $Q^{*}$ distribution shown for comparative purposes.

Figure 10: Temporal variation in normalized total, $q_{b}{ }^{*}$, and fractional, $q_{b i}{ }^{*}$, bed-load transport rates for BM sediment runs with HY1_2 hydrograph conditions (see Table 2).

Figure 11: Temporal variation in bed-load mobility parameter, $\psi_{i}$, for (a) UM and (b) BM sediment runs with HY1_1 hydrograph conditions (see Table 2).

Figure 12: Variation of fractional bed-load yield ratios, $\chi_{i}$, for BM and UM sediment mixtures for (a) HY1_1 hydrograph flow conditions (i.e. varying $\eta$ ) and (b) individual sediment fraction sizes $d(\mathrm{~mm})$ under equivalent steady (ES) flow conditions (see Table 2).

Figure 13: Variation of relative transport yield ratios, $\zeta$ and $\zeta_{i}$, for UM and BM sediment mixtures under (a) - (c) varying hydrograph shape, $\eta$, and (d) varying unsteadiness, $\Gamma_{H G}$.

Figure 14: Variation in normalized bed-load yields, $W_{t}^{*}$, for UM and BM sediments with (a) hydrograph shape, $\eta$, (b) unsteadiness, $\Gamma_{H G}$, and water work, $W_{k}$. Part (c) compares yields obtained with the HY1_1 hydrographs and equivalent steady (ES) flow conditions (Table 2).

\section{Table Captions}

Table 1: Main grain size characteristics and size classifications for the design sediment mixtures.

Table 2: Main experimental variables and derived parameters describing the unsteady flow hydrograph characteristics. 
Figure 1

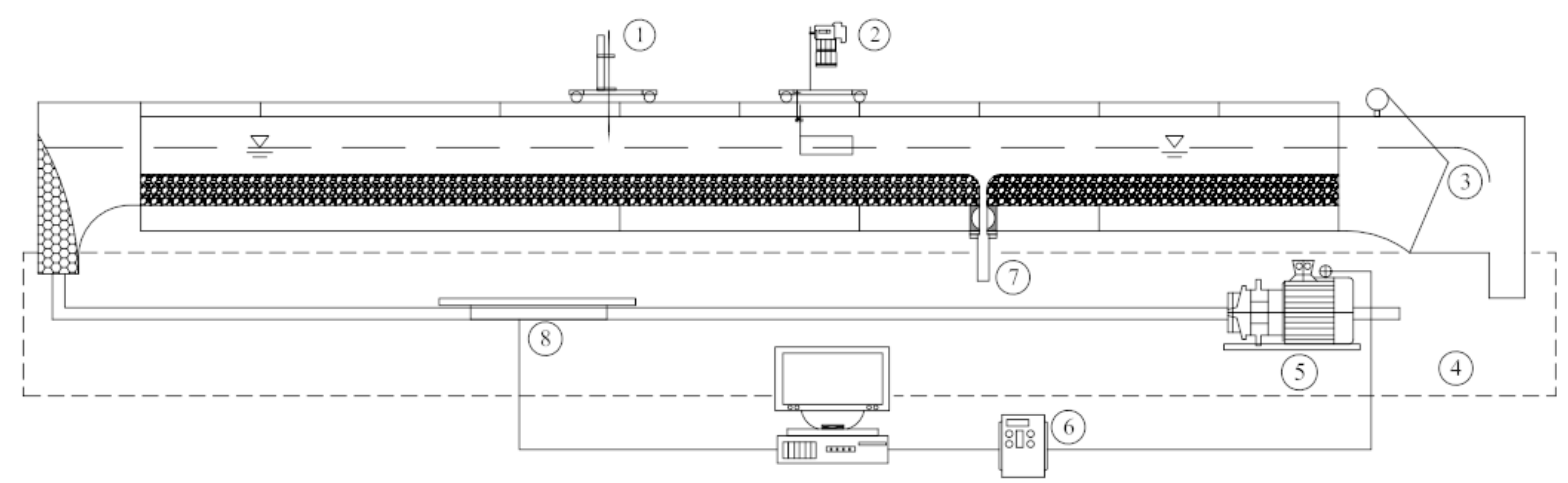

(1) Pointer guage (2) Camera carriage (3) Tail gate (4) Water tank (5) Pump

(6) Frequency inverter (7) Sediment trap (8) Acoustic flow sensor 
Figure 2

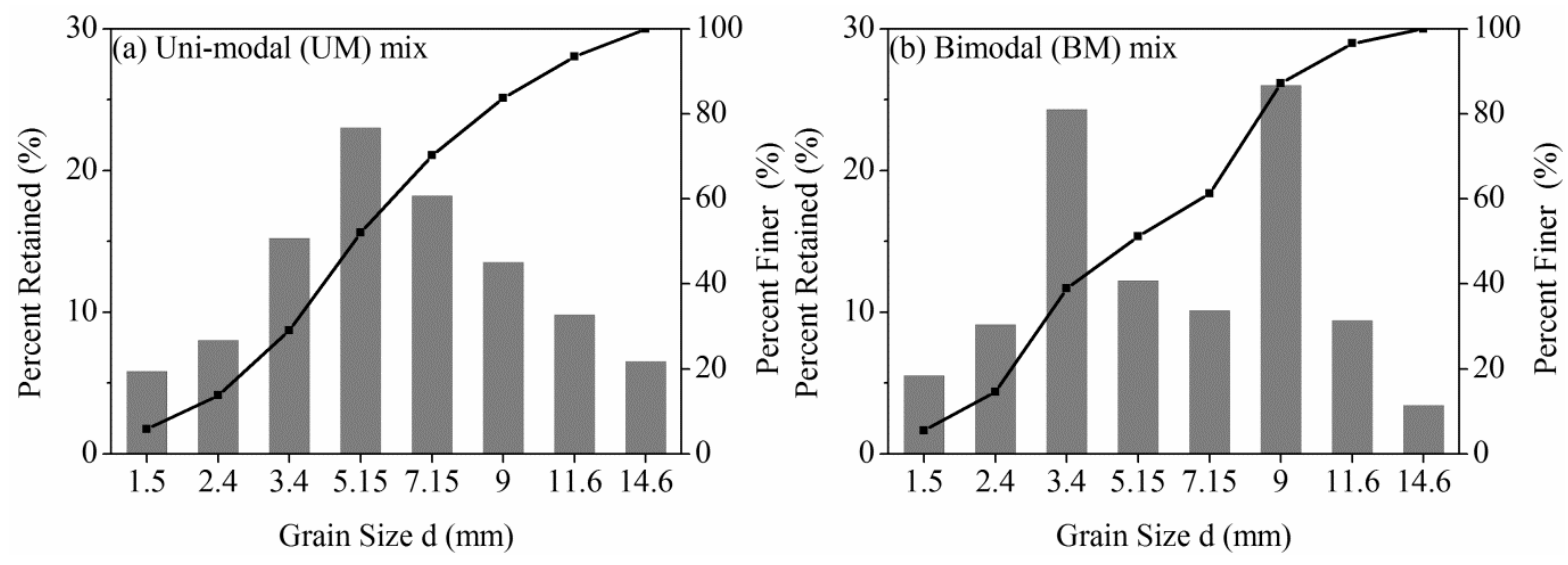


Figure 3

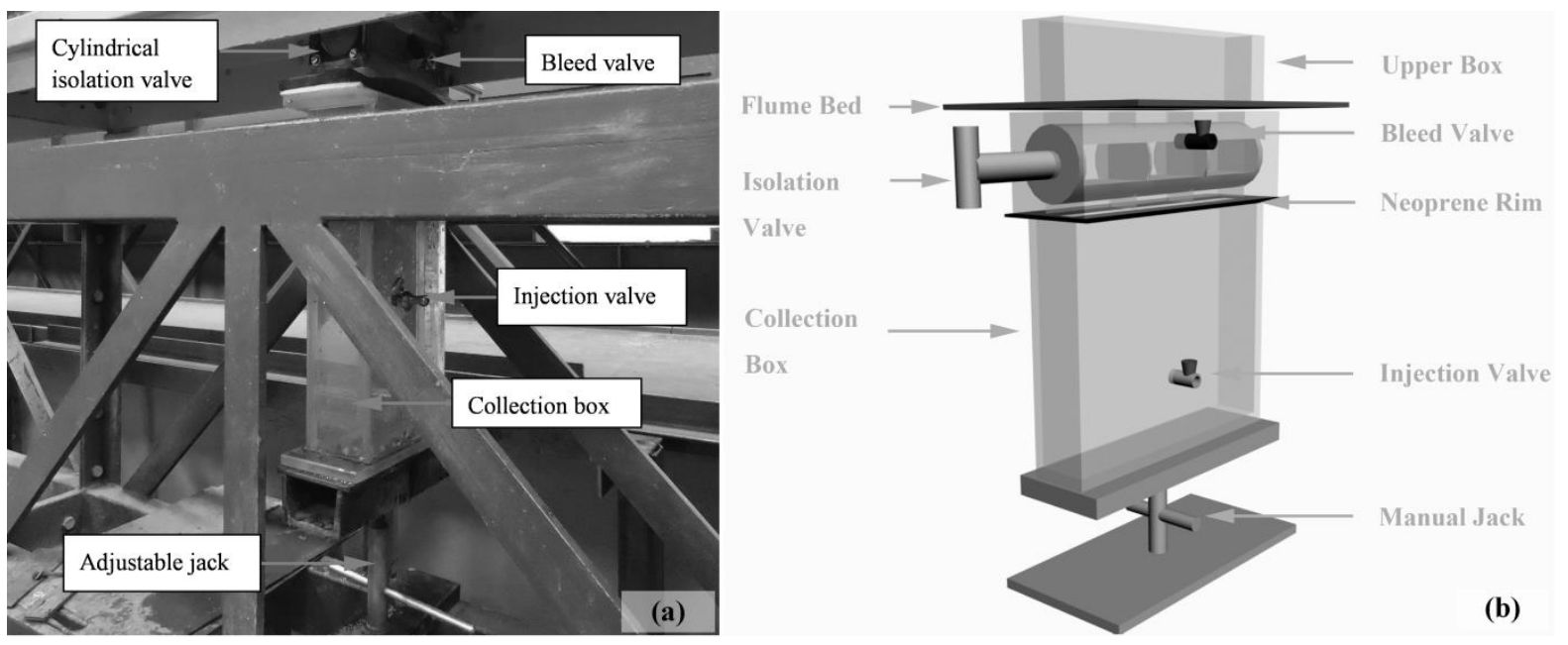


Figure 4
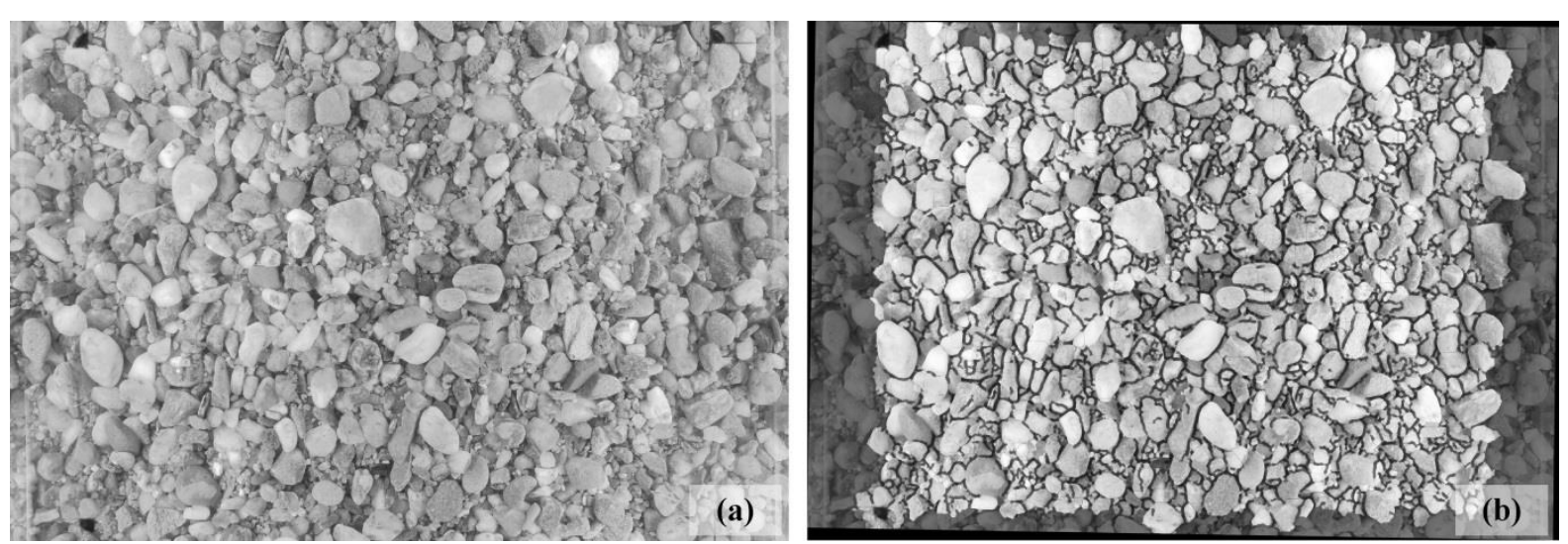
Figure 5
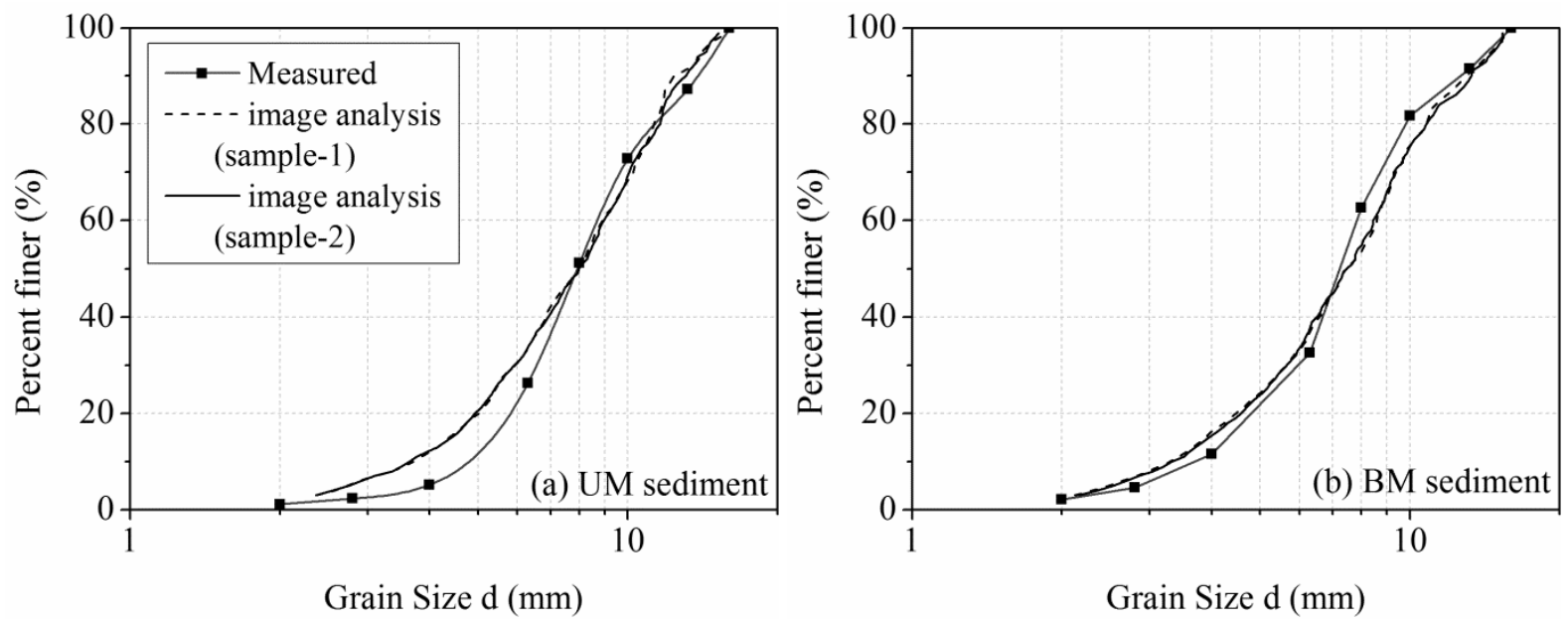
Figure 6
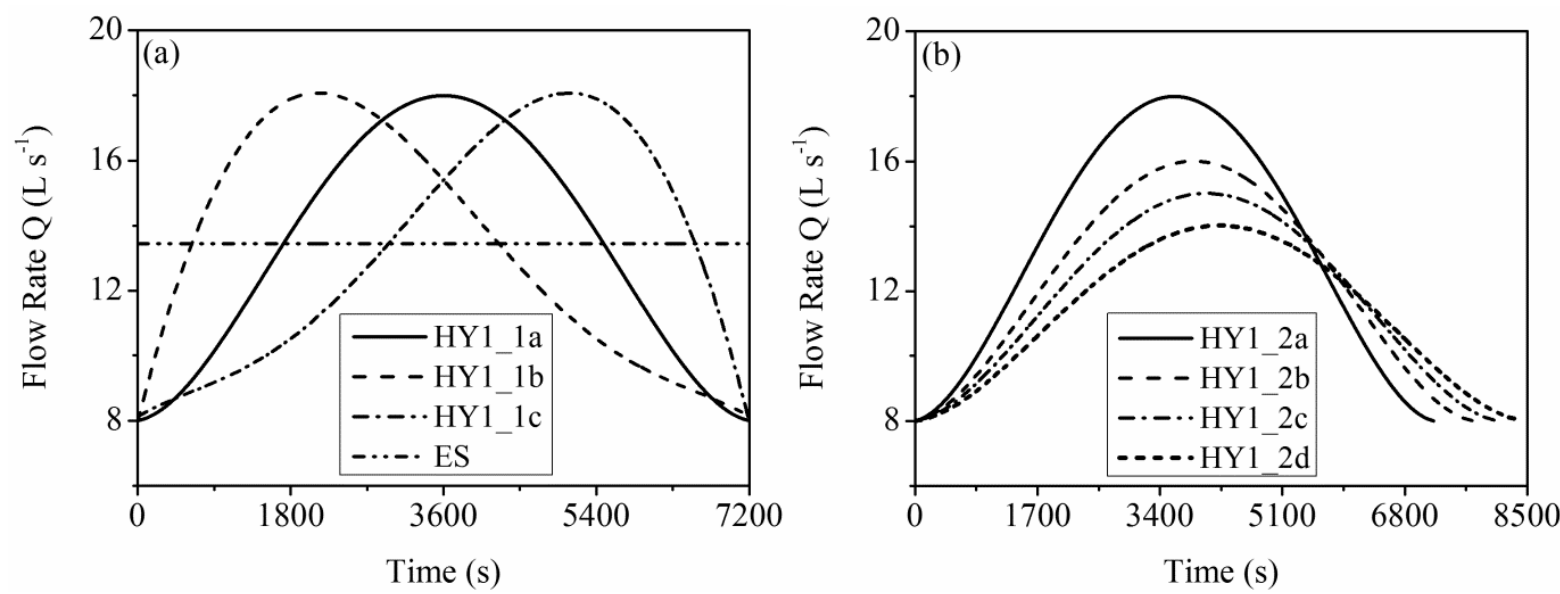
Figure 7
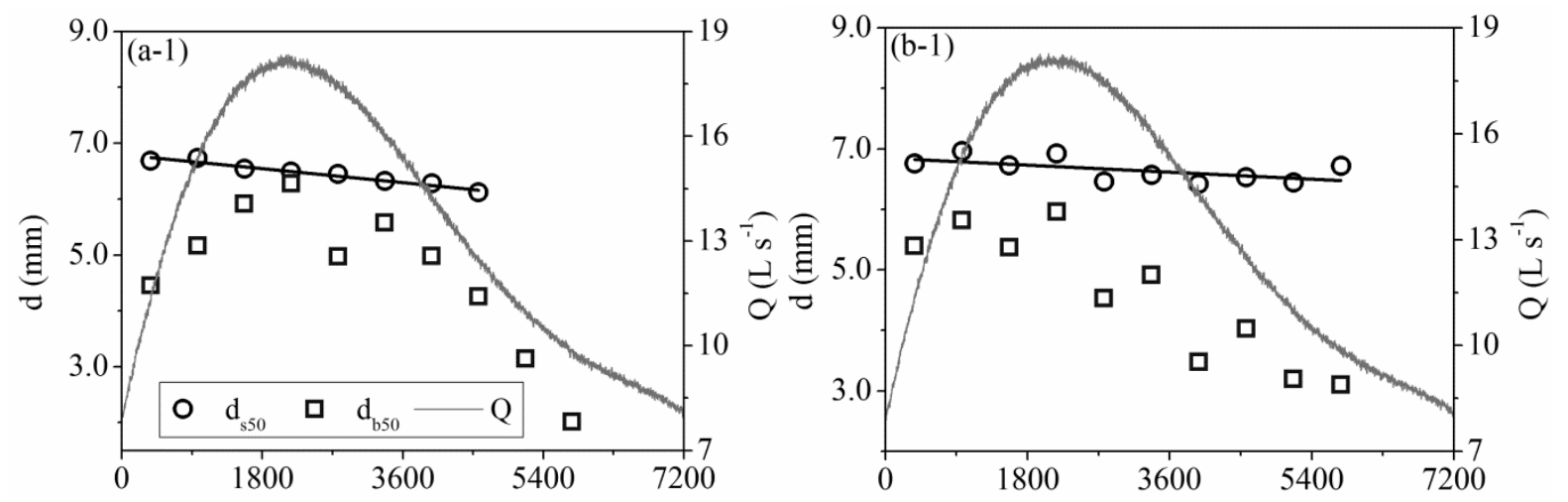

t (s)

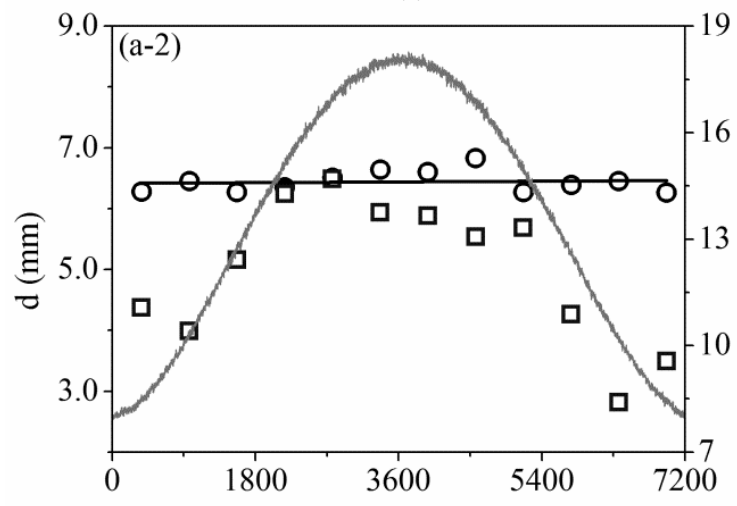

t (s)

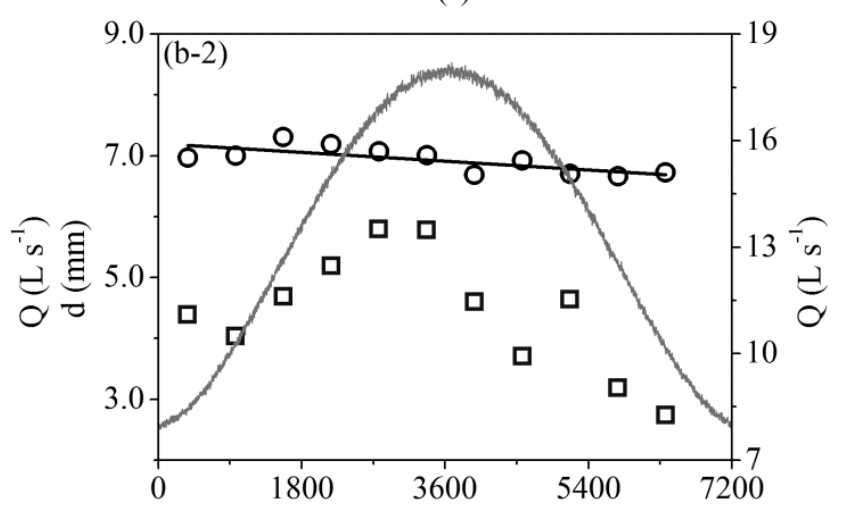

t (s)

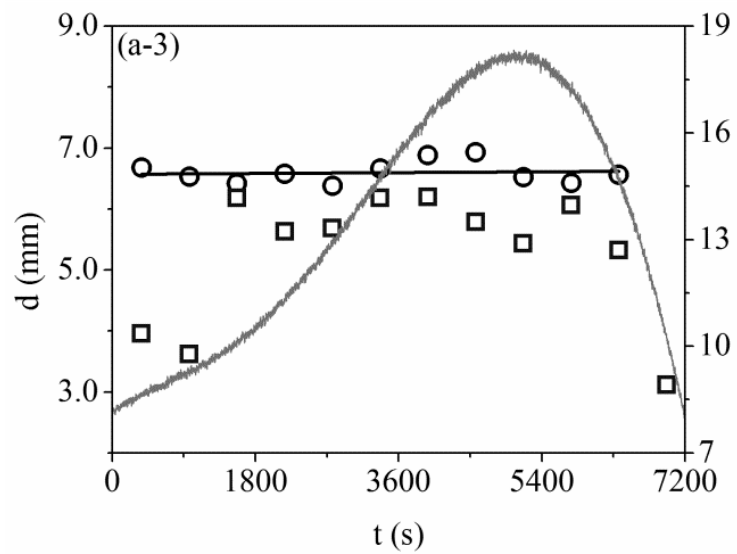

t (s)

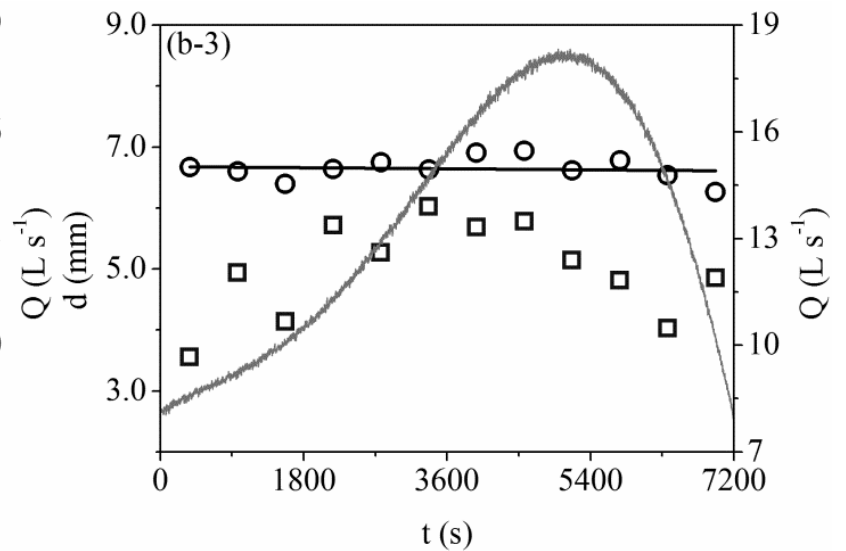


Figure 8
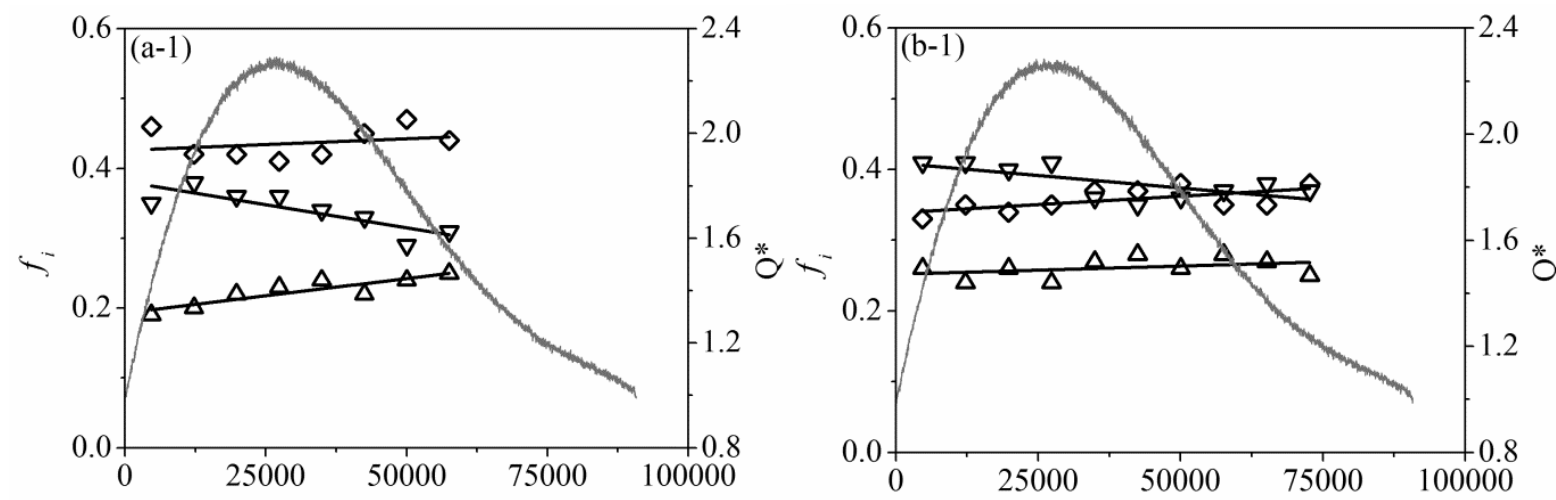

t*

$t^{*}$
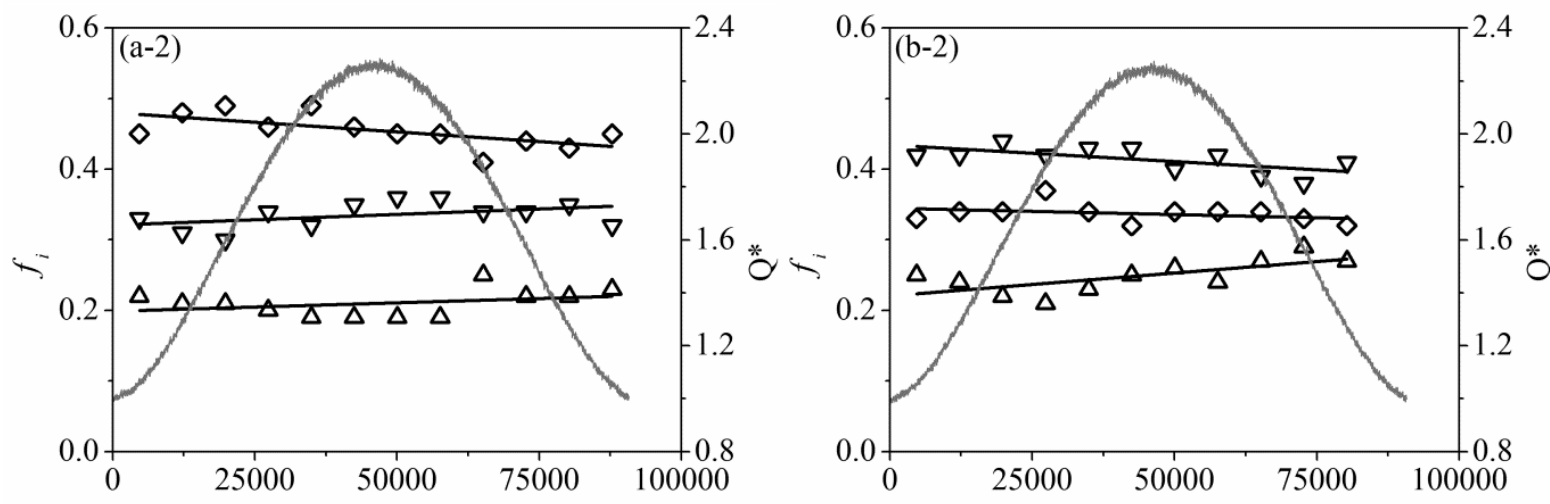

$t^{*}$

$\mathrm{t}^{*}$
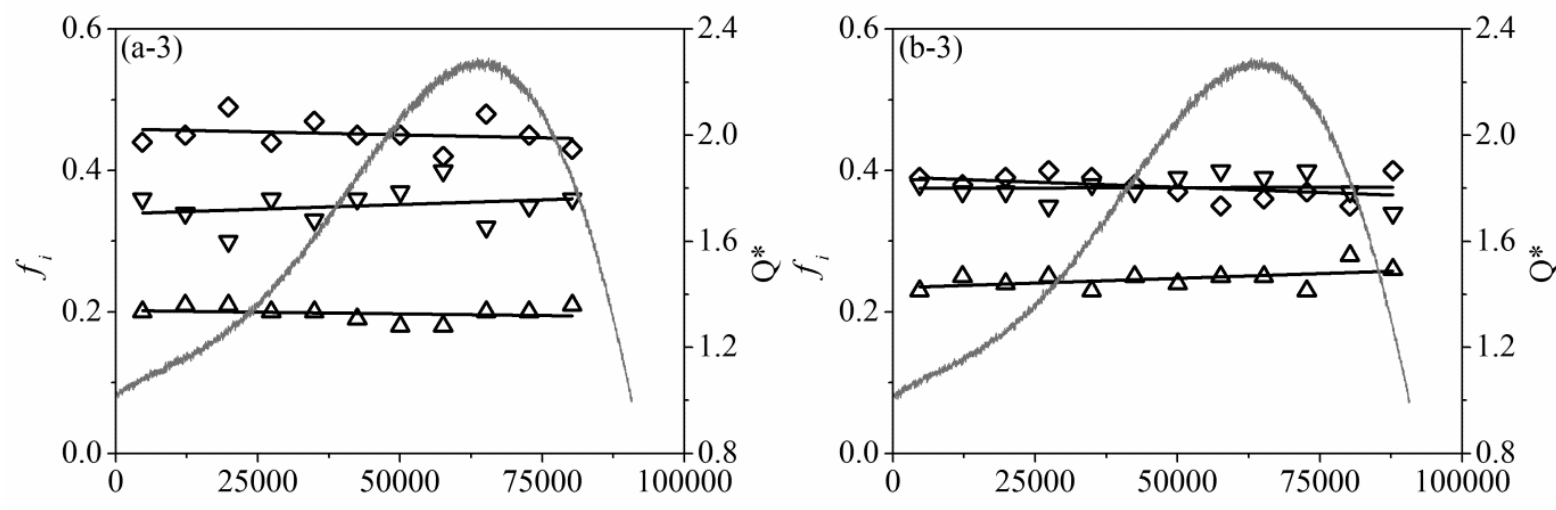

$\Delta$ Fine $\diamond \quad$ Medium $\nabla$ Coarse $-\mathrm{Q}^{*}$ 
Figure 9
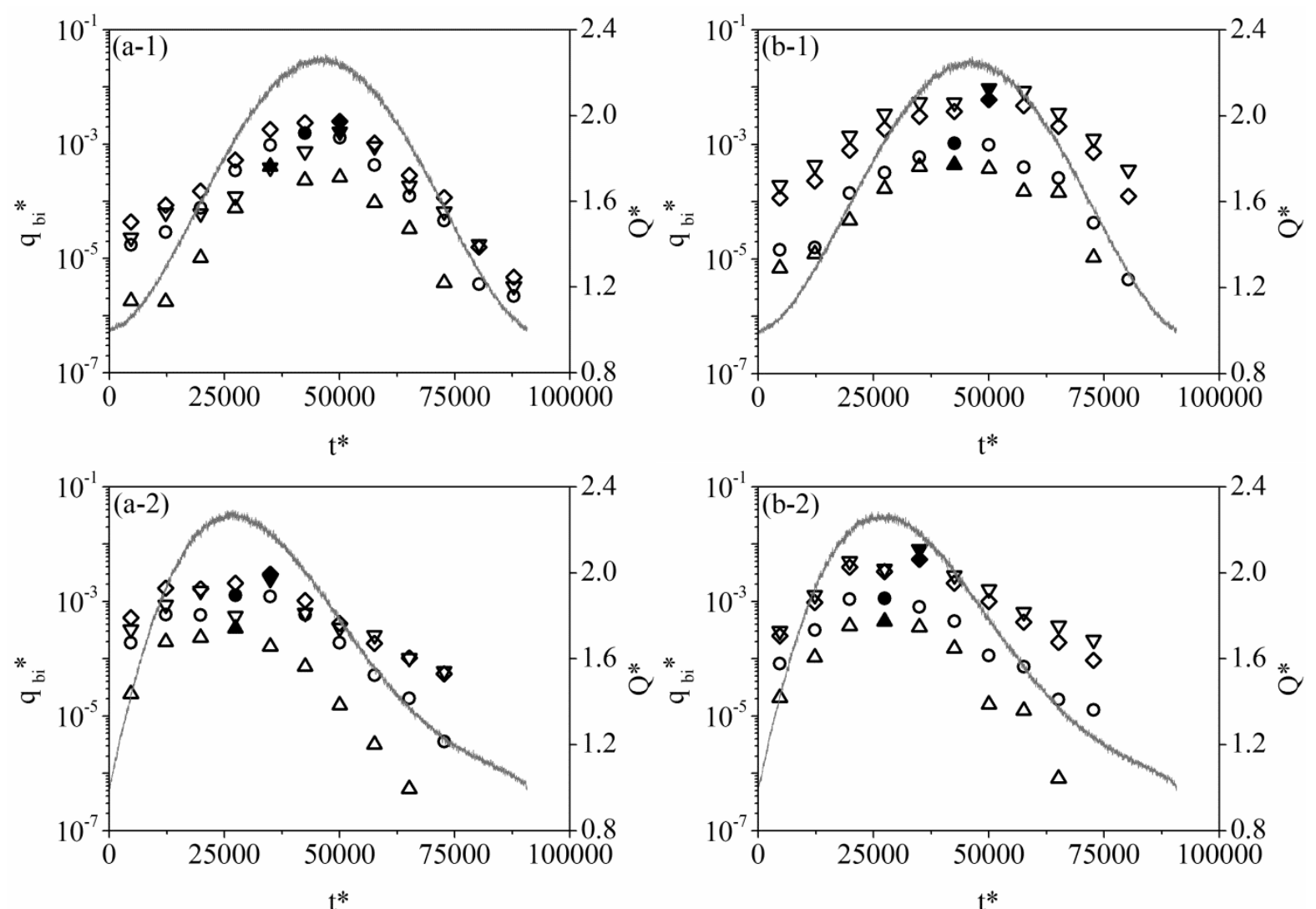

$t^{*}$

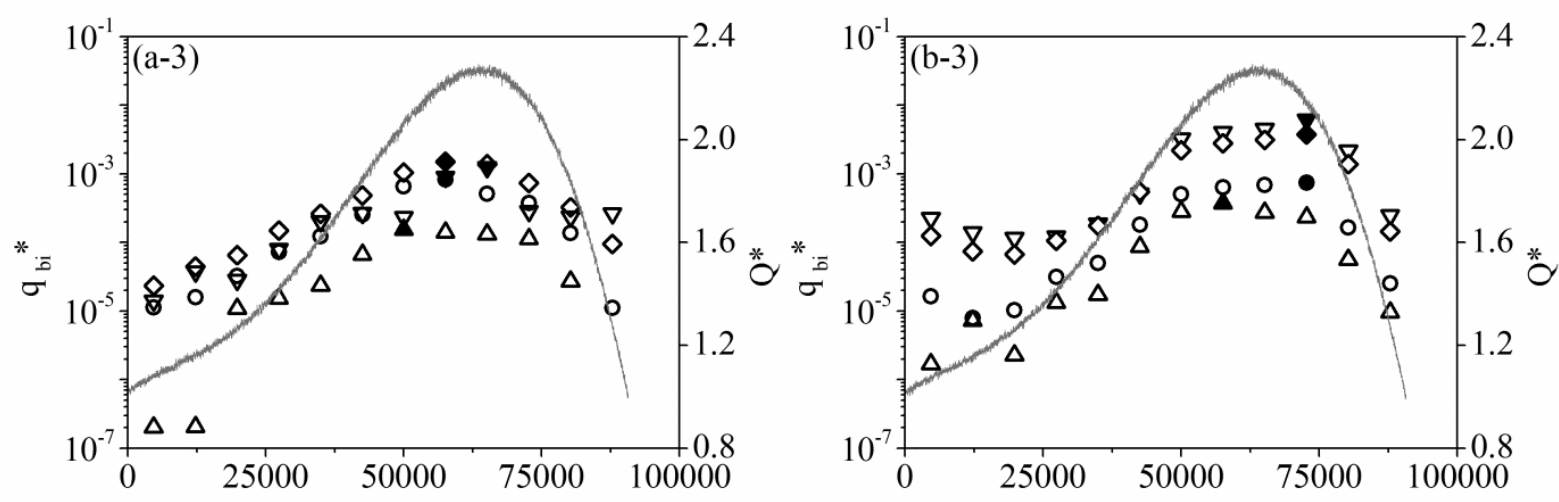

\begin{tabular}{|llllllllll}
\multicolumn{1}{l}{$\mathrm{t}^{*}$} & & & & & & & & & $\mathrm{t}$ \\
$\diamond$ & Total & $\boldsymbol{\nabla}$ & Fine & $\circ$ & Medium & $\Delta$ & Coarse & \\
$\bullet$ & Peak & $\boldsymbol{\nabla}$ & Peak & $\bullet$ & Peak & $\boldsymbol{\Delta}$ & Peak & $\mathrm{Q}^{*}$
\end{tabular}


Figure 10
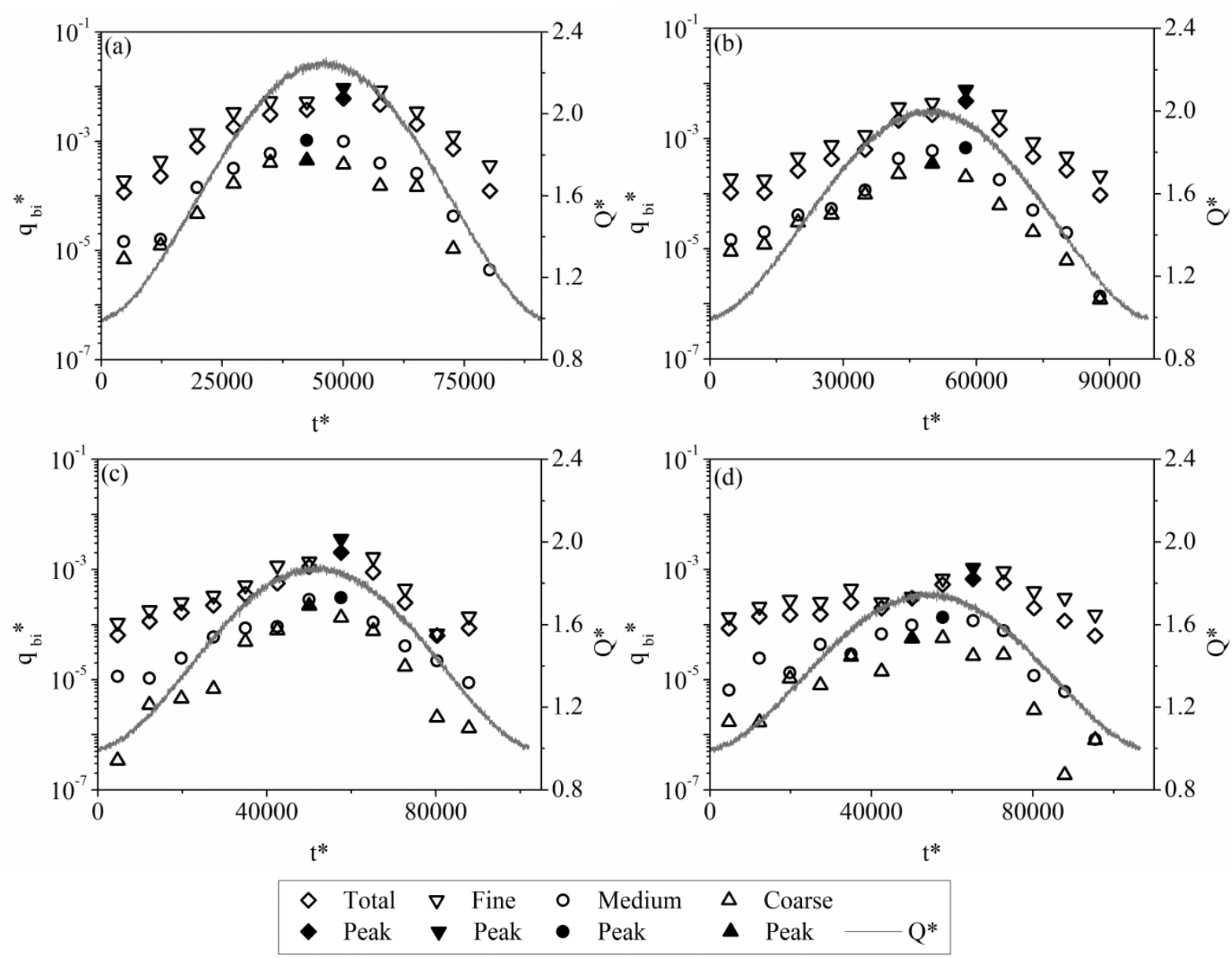
Figure 11
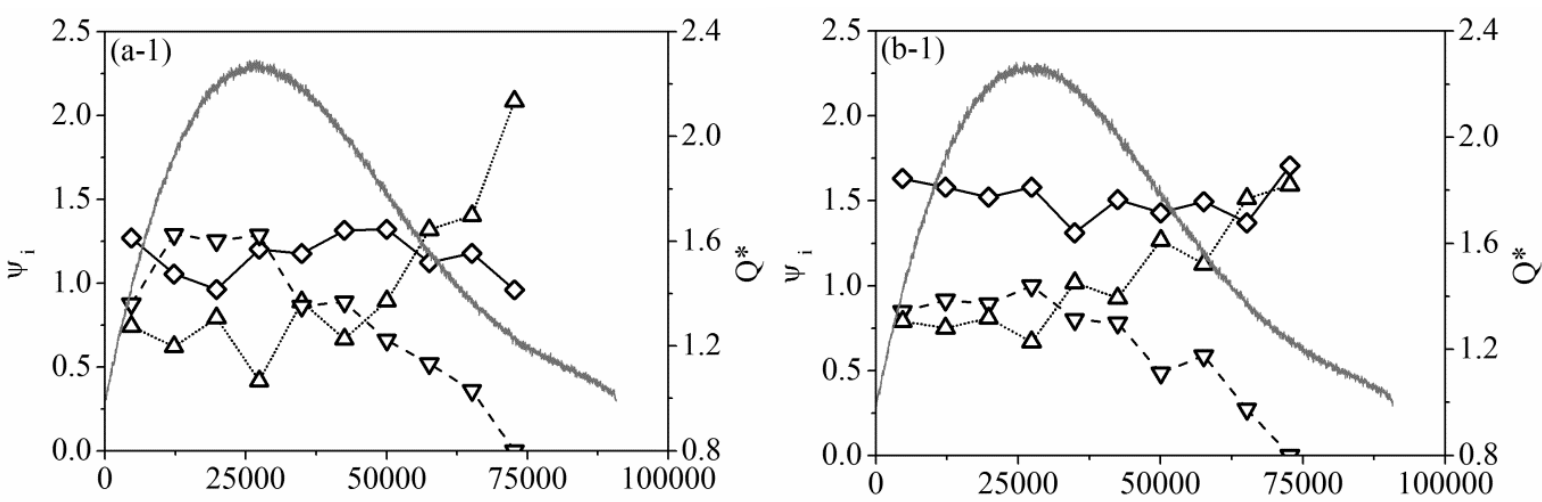

t*

t*
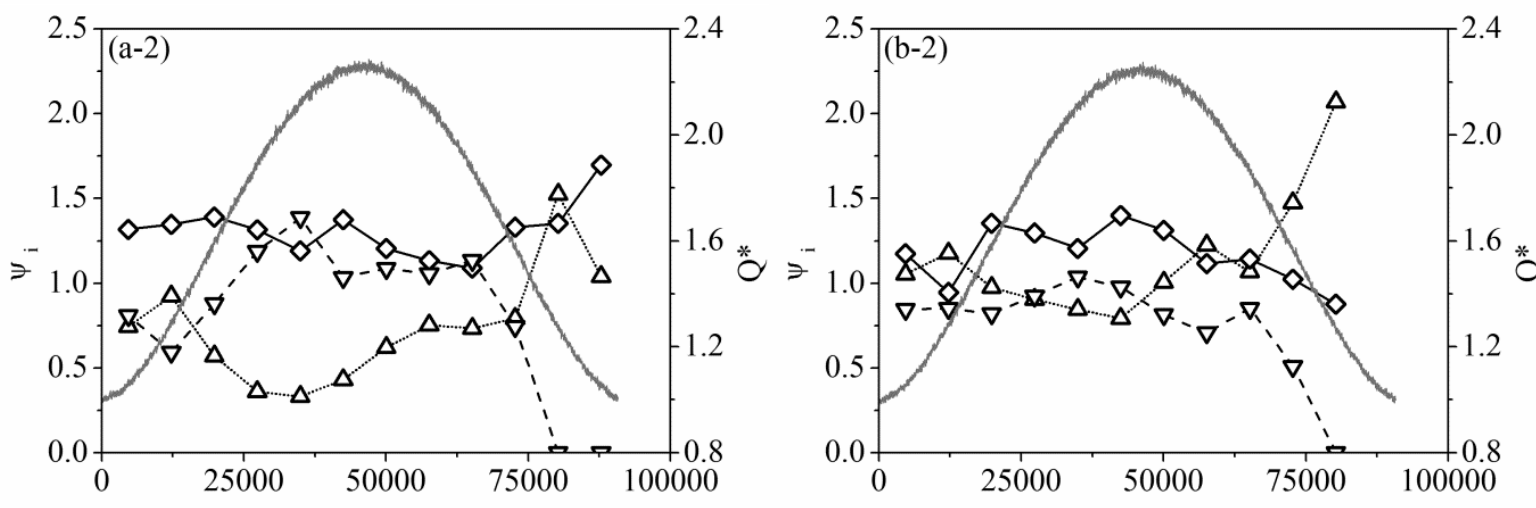

$t^{*}$

t*
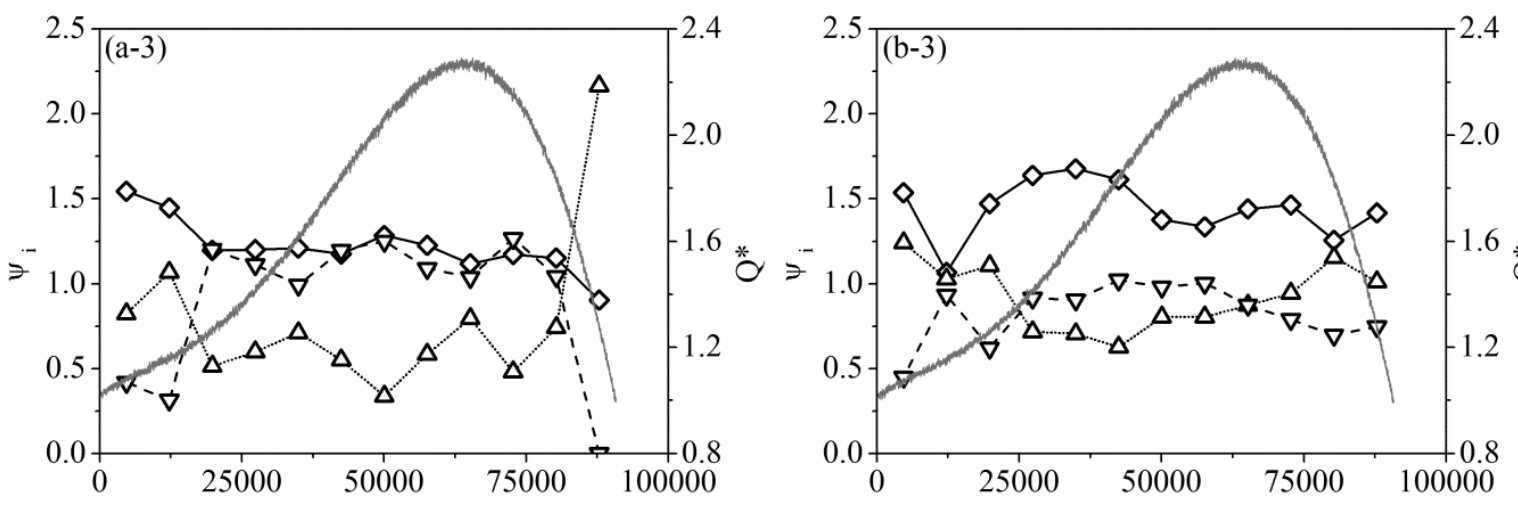

2.0

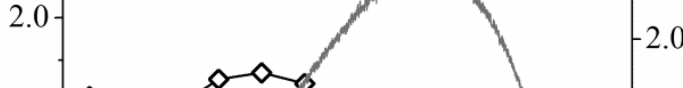

$=1.0-4.5 \%$
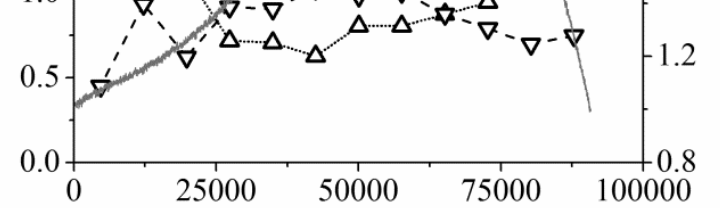

t* t* 
Figure 12
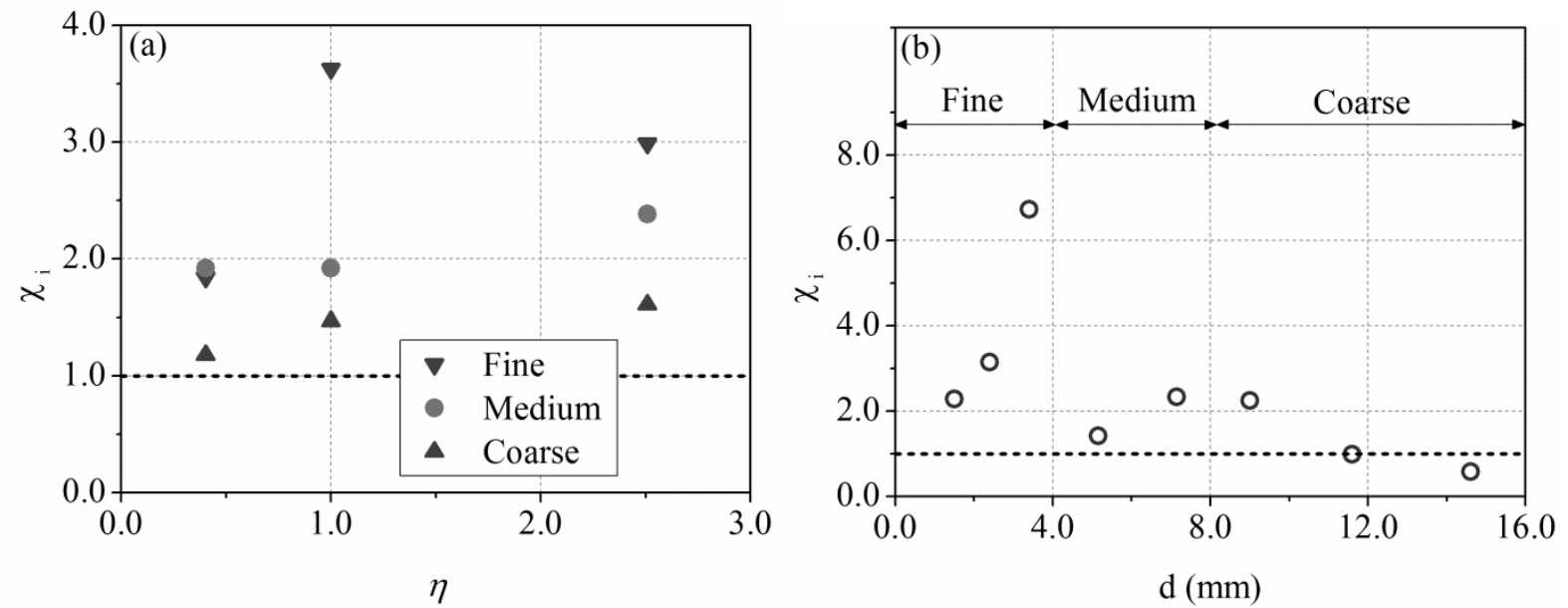
Figure 13
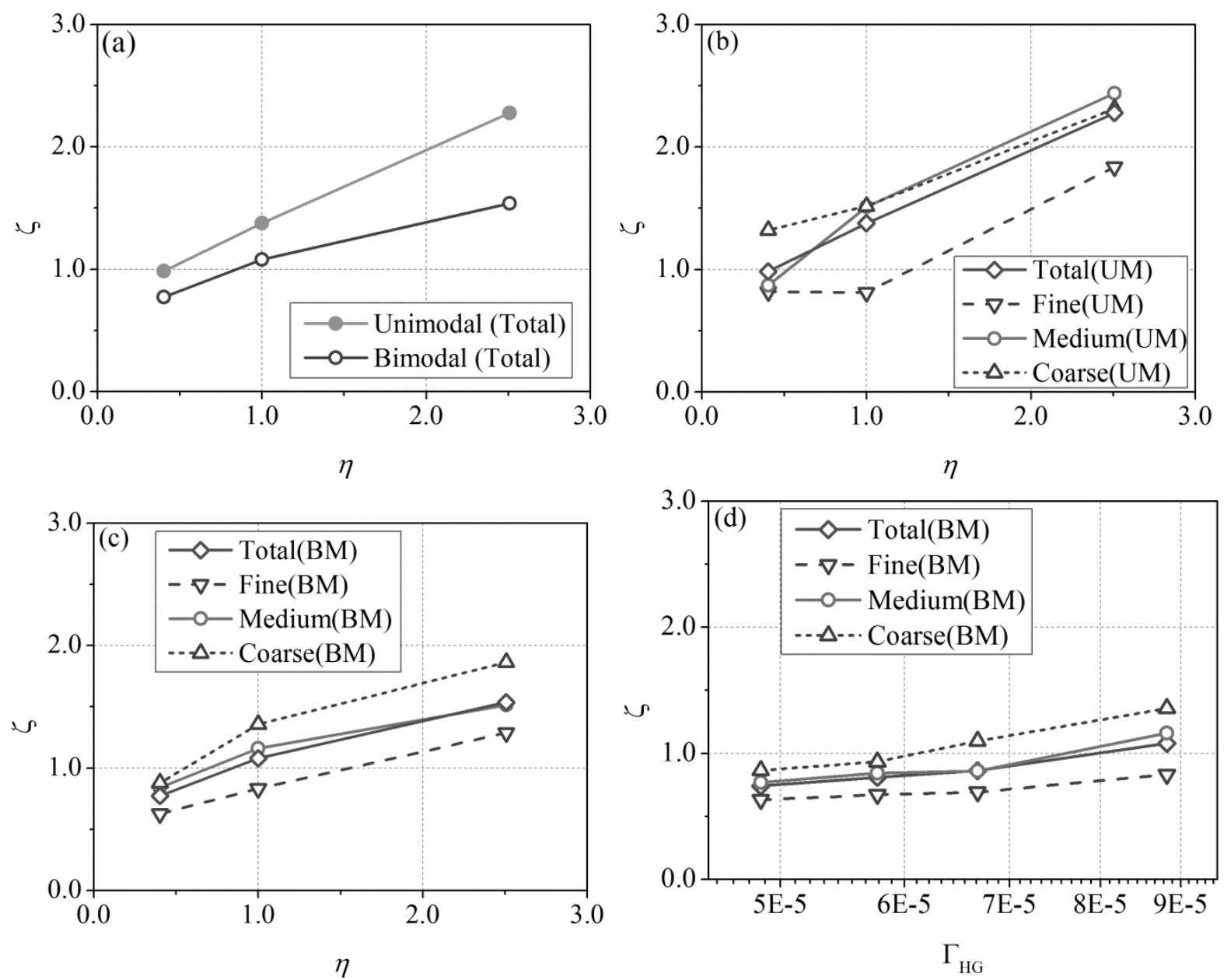
Figure 14

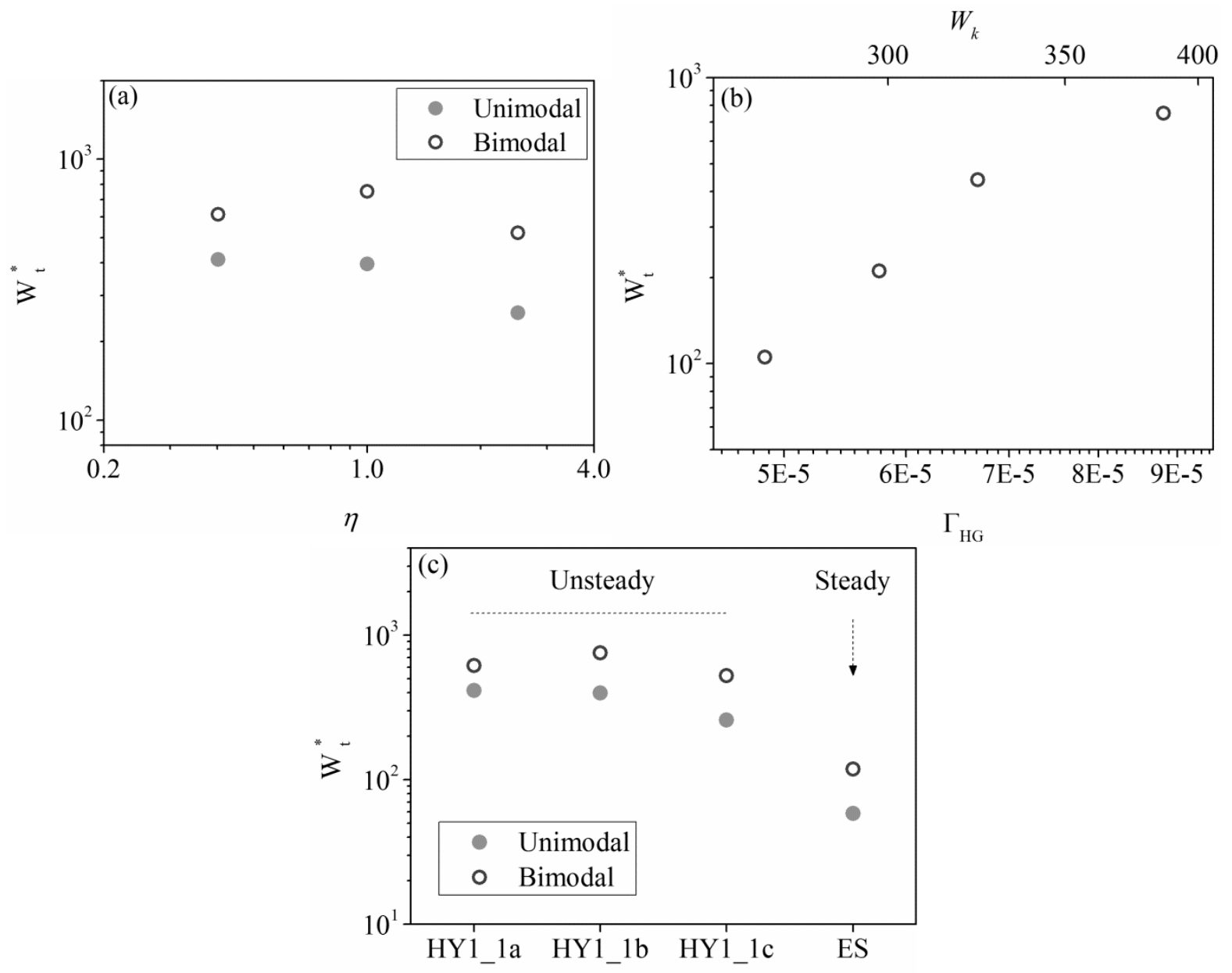

Flow Conditions 
Table 1

\begin{tabular}{ccccccccc}
\hline Design & \multicolumn{3}{c}{ Grain Size $(\mathrm{mm})$} & \multicolumn{3}{c}{ Size classes $(\mathrm{mm})$} \\
\cline { 2 - 5 } Mixture & $d_{16}$ & $d_{50}$ & $d_{84}$ & $d_{90}$ & & Fine & Medium & Coarse \\
\hline UM & 2.54 & 5.0 & 9.08 & 10.67 & 1.89 & $1.0-4.0$ & $4.0-8.0$ & $>8.0$ \\
BM & 2.46 & 5.0 & 8.77 & 9.77 & 1.89 & $1.0-4.0$ & $4.0-8.0$ & $>8.0$ \\
\hline
\end{tabular}


Table 2

\begin{tabular}{|c|c|c|c|c|c|c|c|c|c|c|c|c|}
\hline Run No. & $\begin{array}{c}Q_{0} \\
\left(1 \mathrm{~s} \mathrm{~s}^{-1}\right)\end{array}$ & $\begin{array}{l}Q_{\text {peak }} \\
\left(1 \mathrm{~s}^{-1}\right)\end{array}$ & $\begin{array}{c}h_{0} \\
(\mathrm{~m})\end{array}$ & $\begin{array}{c}h_{p} \\
(\mathrm{~m})\end{array}$ & $\begin{array}{c}u_{* b} \\
\left(\mathrm{~m} \mathrm{~s}^{-1}\right)\end{array}$ & $\begin{array}{l}T_{h} \\
(\mathrm{~s})\end{array}$ & $\begin{array}{l}T_{r} \\
\text { (s) }\end{array}$ & $\begin{array}{c}T_{f} \\
(\mathrm{~s})\end{array}$ & $\begin{array}{l}V_{h}^{+} \\
\left(\mathrm{m}^{3}\right)\end{array}$ & $\Gamma_{H G}$ & $W_{k}$ & $\eta$ \\
\hline HY1_1a $a^{1,2}$ & 8.0 & 18.0 & 0.046 & 0.080 & 0.0535 & 7200 & 3600 & 3600 & 39.26 & 8.82E-05 & 392.80 & 1.00 \\
\hline HY1_1b $b^{1,2}$ & 8.0 & 18.0 & 0.046 & 0.080 & 0.0535 & 7200 & 2058 & 5142 & 39.26 & $8.82 \mathrm{E}-05$ & 392.80 & 0.40 \\
\hline HY1_1 $c^{1,2}$ & 8.0 & 18.0 & 0.046 & 0.080 & 0.0535 & 7200 & 5142 & 2058 & 39.26 & 8.82E-05 & 392.80 & 2.50 \\
\hline HY1_2a ${ }^{2}$ & 8.0 & 18.0 & 0.046 & 0.080 & 0.0535 & 7200 & 3600 & 3600 & 39.26 & 8.82E-05 & 392.80 & 1.00 \\
\hline HY1_2b ${ }^{2}$ & 8.0 & 16.0 & 0.046 & 0.074 & 0.0535 & 7740 & 3870 & 3870 & 33.82 & $6.76 \mathrm{E}-05$ & 338.38 & 1.00 \\
\hline HY1_2 $c^{2}$ & 8.0 & 15.0 & 0.046 & 0.071 & 0.0535 & 8100 & 4050 & 4050 & 31.00 & $5.76 \mathrm{E}-05$ & 310.23 & 1.00 \\
\hline HY1_2d ${ }^{2}$ & 8.0 & 14.0 & 0.046 & 0.068 & 0.0535 & 8460 & 4230 & 4230 & 27.80 & $4.86 \mathrm{E}-05$ & 278.18 & 1.00 \\
\hline $\mathrm{ES}^{1,2}$ & 8.0 & 13.45 & 0.046 & 0.065 & 0.0535 & 7200 & - & - & 39.26 & - & 392.80 & - \\
\hline
\end{tabular}

${ }^{1}$ Uni-modal (UM) sediment mixture; ${ }^{2}$ bimodal (BM) sediment mixture (see Figure 2, Table 1).

${ }^{+}$Total hydrograph volumes excluding base (antecedent) flow contribution. 